\title{
Assessing the influence of local neural activity on global connectivity fluctuations: Application to human intracranial EEG during a cognitive task
}

\author{
Manel Vila-Vidal*a, Mariam Khawaja ${ }^{\mathrm{b}}$, Mar Carreño ${ }^{\mathrm{b}, \mathrm{c}}$, Pedro \\ Roldán $^{\mathrm{d}}$, Jordi Rumià ${ }^{\mathrm{d}}$, Antonio Donaire ${ }^{\mathrm{b}, \mathrm{c}, \mathrm{e}}$, Gustavo Deco ${ }^{1, \mathrm{a}, \mathrm{f}}$, and \\ Adrià Tauste Campo ${ }^{\dagger 1, a, g}$ \\ ${ }^{1}$ These authors jointly supervised this work \\ ${ }^{a}$ Department of Information and Communication Technologies, Universitat Pompeu Fabra, \\ Barcelona, Spain \\ bEpilepsy Program, Hospital Clínic, Barcelona, Spain \\ ${ }^{\mathrm{c}}$ Institut d'Investigacions Biomèdiques August Pi i Sunyer (IDIBAPS), Barcelona, Spain \\ dEpilepsy Program, Neurosurgery, Hospital Clínic, Barcelona, Spain \\ ${ }^{e}$ CIBERBBN, Networking Centre on Bioengineering, Biomaterials and Nanomedicine, \\ Barcelona, Spain \\ f Institució Catalana de Recerca i Estudis Avançats, Barcelona, Spain \\ gDepartment of Applied Physics, Universitat Politècnica de Catalunya, Barcelona, Spain
}

\begin{abstract}
Cognitive-relevant information is processed by different brain areas that cooperate to eventually produce a response. The relationship between local activity and global brain states during such processes, however, remains for the most part unexplored. To address this question, we designed a simple face-recognition task performed in drug-resistant epileptic patients with intracranial EEG. Based on our observations, we developed a novel analytical framework (named "local-global" framework) to statistically correlate the brain activity in every recorded gray-matter region with the widespread connectivity functions as proxy to assess the level of influence of local neural activations into the brain's global state during cognition. The application of our analysis to the data from two subjects was able to detect the local activity in task-relevant brain areas including the primary visual and motor cortices. Despite substantial differences in the recorded regions of each subject, the connectivity functions consistently showed a significant global desynchronization occurring a few hundred milliseconds
\end{abstract}

${ }^{*}$ Corresponding author. Address: Center for Brain and Cognition, Department of Information and Communication Technologies, Universitat Pompeu Fabra, 08005, Barcelona, Spain. E-mail: m@vila-vidal.com

${ }^{\dagger}$ Corresponding author. Address: Center for Brain and Cognition, Department of Information and Communication Technologies, Universitat Pompeu Fabra, 08005, Barcelona, Spain. E-mail: adria.tauste@upc.edu 

made available under aCC-BY-NC-ND 4.0 International license.

after the stimulus onset. In this context, the local-global framework revealed that the reported desynchronization was better explained by the local activity of brain areas involved in face information processing, providing evidence that the global measures might be a novel signature of functional brain activity reorganization taking place when a stimulus is processed in a task context.

Keywords: intracranial EEG, local activity, spectral estimation, global connectivity, cognitive task 


\section{Introduction}

Human cognition implies the contribution of different brain areas that interact to process incoming information and eventually produce a response. A classical localist approach in cognitive neuroscience has attempted to assign cognitive functions to specific brain areas and understand their role at different stages of the cognitive process. This approach has proven successful in partially explaining a number of cognitive processes such as attention, memory or decision making with different recording modalities (Hubel and Wiesel, 1959; Brunel and Wang, 2001; Wang, 2002; Deco and Rolls, 2005; Kahana, 2006; Lachaux et al., 2012). In sharp contrast, more recent studies have taken a globalist approach, focusing on global states that can be measured by means of statistical dependencies across the whole brain (Sporns et al., 2005; Axmacher et al., 2008; Bressler and Menon, 2010; Palva et al., 2010; Bassett et al., 2011; Wang et al., 2015; Deco et al., 2015; Cruzat et al., 2018). Yet, a fundamental question linking the two perspectives remains for the most part unaddressed: how does the processing of cognitive-relevant information in each functionally involved brain area influence the brain's global state?

To tackle this question, we designed a simple face-recognition paradigm that drug-resistant epileptic patients conducted during the pre-surgical intracranial monitoring period (Munari and Bancaud, 1985; Kahane et al., 2003; Lachaux et al., 2003; Engel et al., 2005). During this procedure, the intracranial activity of up to 200 electrode contacts in varied regions from cortical and subcortical regions is simultaneously recorded, which sheds light into the mechanisms of neural activity associated with consciously perceiving and reporting a visual stimulus. In particular, we collected intracranial EEG data (also often referred in the literature as local field potentials, LFP) from depth electrodes stereotactically implanted for pre-surgical diagnosis in 2 drug-resistant epilepsy patients while they were performing the cognitive task.

Inspired by the recorded data, we developed and systematized a methodological pipeline integrating neuroanatomic information, clinical reports, signal processing functions and statistical analysis, with the aim to localize and quantify time-varying human neural activity in the context of a cognitive task. High-frequency LFP power is known to display high correlation with spiking activity of local neuronal assemblies (Hipp et al., 2012; Pesaran et al., 2018). Based on these results, we used LFP high-frequency power activations as proxy for locally generated activity. Yet, the specific frequency range of such activity can vary depending on the type of LFP recording technique. To avoid making further assumptions, we took a data-driven approach and defined the range depending on the observed activations. In order to characterize global network states, we resorted to functional connectivity analysis. Activity in the beta band and below is known to display long-range coher- 
ence and is thought to have a more widespread origin (Pesaran et al., 2018), reflecting possible concurrent inputs or more global states. Based on these premises, we defined two independent functions in the low-frequency range to measure the brain sites' connectivity consistency across time and across trials. In addition, we took advantage of the referential montage to capture these global cofluctuations, as suggested by previous literature (Tauste Campo et al., 2018).

As a result of our work, we propose a novel data-analysis framework (named "local-global" framework) that statistically correlates the brain activity in every gray-matter recording site with the predefined connectivity functions to assess the level of influence of local neural activations into the brain's global connectivity during cognition. We applied this framework to the data gathered from two epileptic patients, detecting local activity in task-relevant brain areas that correlated with global cofluctuations.

\section{Methods}

\section{$2.1 \quad$ Ethics statement}

The study was conducted in accordance with the Declaration of Helsinki and informed consent was explicitly obtained from all participants prior to the recordings and the performance of the tasks. All diagnostic, surgical and experimental procedures have been previously approved by The Clinical Ethical Committee of Hospital Clínic (Barcelona, Spain). In particular, the specific proposal to run the cognitive experiments for this study was approved in March 2020 under the code number HCB/2020/0182.

\subsection{Participants and behavioural task}

Intraranial EEG recordings during performance of certain tasks were acquired in 2 subjects with pharmacoresistant epilepsy during the diagnostic monitoring period in Hospital Clínic (Barcelona, Spain). Details on patients' demographic information is given in Table 1. The two participants had normal or corrected-to-normal vision. The task was designed to characterize brain responses to static face recognition. Stimulus presentation was designed and responses were collected using Psychtoolbox 3 for Matlab.

Subjects viewed $N$ (64 and 71 for subjects 1 and 2, respectively) face images of different identities on a laptop screen. Approximately one half of these images were familiar faces and the remaining half were images from people that the subject was unlikely to know. Familiar faces were selected after a short interview with the subject in which they introduced themselves and talked about their hobbies and interests. Potentially unfamiliar faces were extracted from an extensive database and were selected according to the age, country and background of the subject. Face images had a resolution of 
160x160 pixels, and were presented at the centre of the screen framed by thin lines that randomly changed their color (green and red) from image to image presentation to help the subject maintain attention. Each trial (Fig. 1A) started with a pre-stimulus blank screen that lasted ITI $=0.5 \mathrm{~ms}$ (inter-trial interval), then a face image was presented during time $T_{\text {stim }}(1$ and $0.9 \mathrm{~s}$ for subjects 1 and 2, respectively). Subjects were instructed to pay attention to the presented face image and then, after the face went off the screen, to respond during a maximum timeout of $T_{\text {out }}(10$ and $3 \mathrm{~s}$ for subjects 1 and 2, respectively) whether they recognized the specific person by pressing the trigger button of a joystick with the right hand. Either after pressing the joystick or when the maximum response timeout had elapsed, the next trial started as stated above. The parameters were adjusted depending on cognitive and experimental constraints associated with each subject. This task lasted in total between 5 and 12 minutes.

Subject 2 experienced psychotic symptoms later on during the day of the task. We therefore decided to disregard all behavioral responses (recognized vs non-recognized) from our analysis in this subject. Across the whole study, we analyzed the data in two different settings: a stimuluspresentation-locked setting (for subjects 1 and 2) and a motor-report-locked setting (subject 2).

\subsection{Data acquisition}

LFPs were recorded using 16 and 7 (subjects 1 and 2, respectively) intracerebral multiple contact Microdeep@ platinum-iridium Depth Electrodes (Dixi Medical, Besançon, France; diameter: $0.8 \mathrm{~mm}$; 5 to 18 contacts, contact length: $2 \mathrm{~mm}$, distance between contacts: $1.5 \mathrm{~mm}$ ) that were stereotactically implanted using frameless stereotaxy, neuronavigation assisted, and intraoperative CT-guided O-Arm and the Vertek articulated passive arm. 175 and 93 contacts were implanted and recorded in subjects 1 and 2, respectively (see Table 1 for details and Fig. 1B for an example of the implantation scheme of subject 1). The decision to implant, the selection of the electrode targets and the implantation duration were entirely made on clinical grounds using the standard procedure (Lachaux et al., 2003; Cardinale et al., 2013). All recordings were obtained using a standard clinical EEG system (XLTEK, subsidiary of Natus Medical) with a $2048 \mathrm{~Hz}$ sampling rate. All signals were referenced to the scalp electrode $\mathrm{CPz}$.

Individual pre- and post-implant T1-weighted MR scans were used to determine contact localizations. MR scans were obtained with a $1.5 \mathrm{~T}$ unit (Magnetom Aera 1.5T; Siemens Medical Systems, Erlangen, Germany) with a specific protocol that included the following sequence: sagittal T1weighted gradient recalled (repetition time [TR] $20 \mathrm{~ms}$, echo time [TE] 7.38 $\mathrm{ms}$, Flip Angle [FA] 20, $1 \mathrm{~mm}$ slice thickness). 


\subsection{Anatomical localization of the SEEG electrode contacts and ROI definition}

Contact anatomical locations were directly identified from the individual subjects' post-implant MRI by visual inspection $\left(\mathrm{MVV}^{1}\right)$, where contacts can be distinguished as dark spherical artifacts (diameter $\approx 3 \mathrm{~mm}$; see Fig. 1B). MRI images were analyzed using the DICOM Viewer Osirix Lite (v.12.0.1). Contact localization within brain structures were therefore obtained with an error of the contact midpoint of approximately $1.5 \mathrm{~mm}$. First, contacts were labelled either as grey matter (GM) or white matter (WM). Previous research suggests that electrical fields generated in GM can be measured by contacts in nearby WM up to $\approx 1 \mathrm{~mm}$ away (Buzsáki et al., 2012; Arnulfo et al., 2015; Narizzano et al., 2017). Based on this assumption, WM contacts lying at a distance below $1 \mathrm{~mm}$ from GM regions with no contact inside were assigned to that region and classified as GM. Contacts lying outside brain tissue or within altered brain tissue according to clinicians (e.g. heterotopias, focal cortical dysplasias) were excluded from the analysis. The electrode contacts lying in the suspected epileptic focus were identified by clinical experts using gold-standard procedures and were also excluded from the study.

GM contact locations were expressed in terms of the Desikan-Killiany (Desikan et al., 2006) brain atlas (34 ROIs per hemisphere), with an extra ROI for the hippocampus, by visually identifying well-defined anatomical landmarks (MVV). Table 2 summarizes the number of contacts and electrodes in each ROI for both subjects. Contacts were also approximately mapped to Brodmann areas, when possible. For further validation, automatic parcellations were created using the post-implant MRI scans and the Free-surfer software, which confirmed manual localizations. GM contacts were also mapped, when possible, to functional ROIs (regions of interest) as usually expressed in the cognitive literature based on fMRI and electrophysiological studies (e.g. V1/V2, DLPFC, VLPFC, M1, PMC), which were also confirmed by a neurologist $\left(\mathrm{MK}^{2}\right)$.

For the purpose of this study, V1/V2 was defined roughly as the primary and/or secondary visual cortices. The DLPFC (dorsolateral prefrontal cortex) was defined roughly as the middle frontal gyrus, the VLPFC (ventrolateral prefrontal cortex) was defined as the inferior frontal gyrus and the superior parts of the pars triangularis, pars orbitalis and pars opercularis. M1 (primary motor cortex) was defined as the precentral gyrus and PMC (premotor cortex) was roughly defined as corresponding to Brodmann area 6 , i.e., as a vertical strip extending from the cingulate sulcus to the lateral sulcus, including caudal portions of the superior frontal and middle frontal gyri, and rostrally bounded by the precentral gyrus. The remaining ROIs

\footnotetext{
${ }^{1}$ Manel Vila-Vidal

${ }^{2}$ Mariam Khawaja
} 
were referred to using its denomination in the Desikan-Killiany atlas. In particular, for this study: suparmarginal gyrus (SMG), insula (I), inferior temporal gyrus (ITG), middle temporal gyrus (MTG), superior temporal gyrus (STG), ventral anterior cingulate cortex (vACC), dorsal anterior cingulate cortex (dACC), and a single posterior cingulate cortex (PCC), under which we grouped the posterior cingulate and the isthmus cingulate.

\subsection{Signal pre-processing}

Besides the contacts mentioned in Section 2.4, we also excluded from the computational analysis contacts displaying highly non-physiological activity. SEEG signals were preprocessed and analyzed using custom-made code in Python 3 based on the Numpy, Scipy and MNE libaries. Signals were analyzed in the referential montage (reference to $\mathrm{CPz}$ ). Prior to the main analysis, they were low-pass filtered with a zero-phase FIR filter with cutoff at $700 \mathrm{~Hz}$ and stopband at $875 \mathrm{~Hz}(175 \mathrm{~Hz}$ transition bandwidth, $-6 \mathrm{~dB}$ supression at 787.5 , maximal ripples in passband $2 \%$ ) to remove aliasing effects. A high-pass zero-phase FIR filter with cutoff at $1 \mathrm{~Hz}$ and stopband at $0 \mathrm{~Hz}(1 \mathrm{~Hz}$ transition bandwidth, $-6 \mathrm{~dB}$ supression at $0.5 \mathrm{~Hz}$, maximal ripples in passband $2 \%$ ) was also applied to remove slow drifts from the SEEG signals. Additionally, we also used a band-stop FIR filter at $50 \mathrm{~Hz}$ and its harmonics to remove the power line interference $(1 \mathrm{~Hz}$ band-stop width, $53 \mathrm{~dB}$ attenuation at center frequency, maximal ripples in passband $2 \%$ ).

In addition, we identified time periods containing widesperad artifacts or interictal epileptic events such as spikes, using the procedure desribed in (Arnulfo et al., 2015). Following this method, we used Morlet wavelets (width $m=7$ ) to obtain a spectral decomposiotion of each signal into 33 logarithmically scaled frequencies from 2 to $512 \mathrm{~Hz}$ in steps of $1 / 4$ octave. Then, we divided the signal envelopes in non-overlapping $250 \mathrm{~ms}$ temporal windows. Corrupted temporal windows were defined as those where at least $10 \%$ of contacts had amplitude envelopes 5 standard deviations above their respective mean amplitude in more than half of the 33 frequency bands.

\subsection{Event-related potentials}

Before conducting the spectral analysis, we analyzed the average signal across trials for a selection of relevant recording sites in each subject. For subject 1, our relevant contact was placed in V1/V2 (deepest electrode contact of the trajectory highlighted in Fig. 1B). To compute the event-related potential (ERP) that was locked to the stimulus-onset and avoid potential noise due the low number of trials, we further low-pass-filtered the data under $70 \mathrm{~Hz}$ (FIR filter with $6 \mathrm{~dB}$ supression at $11.25 \mathrm{~Hz}$, maximal ripples in passband $2 \%$ ). We then epoched the data (-500 ms to $1500 \mathrm{~ms}$ from stim- 
ulus onset), baseline-corrected each epoch (subtraction of the mean across the baseline period, from -500 to $0 \mathrm{~ms}$ ) and extracted the median across trials.

For subject 2, our relevant contact was placed in the left-hemisphere primary motor cortex (M1), approximately at the location of right-hand movement control. In this case, we extracted the ERP in M1 during recognized and non-recognized trials, time-locked to button press or response timeout end, respectively. To compute the ERPs, we low-pass-filtered the data under $70 \mathrm{~Hz}$, epoched the data $(500 \mathrm{~ms}$ before stimulus presentation to $500 \mathrm{~ms}$ after button press or response timeout end, respectively), baselinecorrected each epoch (subtraction of the mean across the baseline period, from -500 to $0 \mathrm{~ms}$ before stimulus presentation), aligned the data to button press or response timeout end, respectively, chunked each epoch from -500 to $500 \mathrm{~ms}$ from behavioural response and extracted the median across trials of each type (recognized vs non-recognized). In this case, we tested differences in the signals across both conditions using the following procedure. Experimental conditions were compared using a Ranksum test at each time point, with a criterion of $P<0.05$ for a minimum of 102 consecutive samples (50 $\mathrm{ms})$.

\section{7 $\quad$ Spectral estimates}

Spectral power was estimated from 4 to $512 \mathrm{~Hz}$ using an adaptive multitaper method based on discrete prolate spheroidal sequences (DPSS, aka. Slepian sequences) (Slepian and Pollak, 1961; Thomson, 1982; Mitra and Pesaran, 1999). For our analysis, we used custom-made code to achieve the highest flexibility in adjusting the temporal and frequency smoothing for each frequency independently. Following this approach, we sought to find the best temporal resolution at lower frequencies, while obtaining more accurate power estimates at typically low SNR (signal-to-noise ratio) higher frequencies, at the expense of temporal and frequency resolution.

As suggested by previous literature (Buzsáki and Draguhn, 2004; Hipp et al., 2012), both the mean frequency and bandwidth of meaningful brain activity typically follow a logarithmic progression. Low-frequency activity (theta, alpha, beta) is thought to be oscillatory, frequency-specific, and less spatially localized, reflecting a sum of different contributions, in particular widespread post-synaptic potentials. On the other hand, high frequency activity (gamma, high-gamma and above) has a broadband profile and is typically thought to reflect locally synchronized neuronal activity. However, the specific frequency range of such activity is not well established and can vary depending on the recording technique.

To consistently capture the specificities of low and high frequency activity, we computed power estimates across 29 logarithmically scaled frequencies $f$ from 4 to $512 \mathrm{~Hz}$ in steps of $1 / 4$ octave, i.e., each frequency was 
obtained by multiplying the previous one by $2^{1 / 4}$. In addition, we adjusted the spectral smoothing parameter to match $3 / 4$ octave, yielding a spectral resolution of $[f-0.34 f, f+0.34 f]$ for each frequency of interest $f$. Regarding the temporal resolution, we followed a different approach for frequencies above and below $32 \mathrm{~Hz}$. For every frequency of interest $f$ below $32 \mathrm{~Hz}$, we adjusted time windows to include 6 cycles of $f$, using shorter windows for larger frequencies. This yielded a temporal smoothing ranging from $t \pm 750$ $\mathrm{ms}$ at $4 \mathrm{~Hz}$ to $t \pm 95 \mathrm{~ms}$ at $32 \mathrm{~Hz}$ around each time point $t$ for our estimates. With this temporal and frequency smoothing, we could use a total of 3 tapers for the spectral estimates at each frequency. In contrast, for frequencies above $32 \mathrm{~Hz}$, we used a fixed temporal smoothing of $\pm 100 \mathrm{~ms}$ around each time point (total temporal smoothing of $200 \mathrm{~ms}$ ), which allowed us to use a greater number of tapers for larger frequencies (from 3 tapers at $32 \mathrm{~Hz}$ to 69 tapers at $512 \mathrm{~Hz}$ ), thus increasing the signal-to-noise ratio of our spectral estimates.

To avoid undesired boundary effects, we first obtained spectral estimates across the whole task period and epoched the data afterwards. For stimulusrelated responses, we considered the epoch comprised from $500 \mathrm{~ms}$ before stimulus presentation to $1500 \mathrm{~ms}$ after stimulus presentation (this includes the stimulus period and a post-stimulus period of at least $500 \mathrm{~ms}$ ). Trial periods containing corrupted time windows as defined in section 2.5 were discarded. For each trial, time-frequency plots were normalized at each scale by the mean power (division by the mean power at that scale) computed during the baseline period (from -400 to -100 ms, to avoid temporal contamination), as done in (Rey et al., 2014). Then, we took the median across all trials to characterize each contact's response to the stimulus.

On the other hand, for motor-related responses (recognized and nonrecognized), we considered the epoch comprised from $500 \mathrm{~ms}$ before stimulus presentation to $500 \mathrm{~ms}$ after button press or response timeout end, respectively. For each trial, time-frequency plots were normalized at each scale by the mean power (division by the mean power at that scale) computed during the baseline period (from -400 to $-100 \mathrm{~ms}$, to avoid temporal contamination). We then aligned the data to button press or response timeout end, respectively, chunked each epoch from -500 to $500 \mathrm{~ms}$ from behavioural response and extracted the median across trials of each type (recognized vs non-recognized).

To validate our assumption that multitaper estimation might better capture local high-frequency activations than other temporally-resolved techniques, we repeated the whole analysis using a wavelet approach, and compared the spectral power estimates. Power estimates were obtained between 4 to $512 \mathrm{~Hz}$ using the same 29 logarithmically scaled frequencies $f$ as before with Morlet wavelets $(m=7)$. We then epoched, normalized and averaged the spectrograms exactly as we did with the multitaper estimates. In addition, we repeated the same analysis filtering the wavelet power estimates 
below $8 \mathrm{~Hz}$ before epoching and averaging, to obtain a similar temporal smoothing than with the continuous multitaper method. See Supplementary Information and Supplementary Fig. S1 for results and discussion.

\subsubsection{Statistical inference of task-related activations}

When assessing stimulus-related activations, the median of the spectrograms obtained by means of multitaper power estimation was computed over all face presentation trials. Following (Rey et al., 2014), we visually identified time-frequency windows of interest (TFOIs) related to the stimulus presentation on the trial-median spectrograms of certain recording sites of interest (for instance, marked with black rectangles in Fig. 2B). We then quantified the strength in the LFP power response in the defined TFOIs using the following non-parametric method. For each TFOI we defined a surrogate baseline TFOI (window spanning the same frequency ranges and time-width of the TFOI centered in the baseline period). For each trial, we extracted the mean power within the TFOI and the surrogate TFOI. We used a Ranksum test to evaluate significance of TFOI mean power against its surrogate baseline.

When assessing motor-related activations locked to the behavioural response (only subject 2), we computed the median of the spectrograms across the two conditions (recognized vs non-recognized) separately. In recognized trial-median spectrograms we visually identified time-frequency windows of interest (TFOIs) and tested the significance of their activations against the baseline period and against the non-recognized trials independently. Significance with respect to baseline was tested using the procedure described in the previous paragraph. Significance with respect to non-recognized trials was tested using a Ranksum test on TFOI mean power values between conditions.

\subsection{Global connectivity variables}

To characterize the global connectivity state we used two complementary connectivity measures. The first measure is the mean functional connectivity $(\mathrm{mFC})$ and characterizes signal temporal cofluctuations across recording sites. The second measure is the mean inter-trial phase-locking value (mITP) and characterizes the global degree of consistency of phase coupling among recording sites across trials (at each time and frequency point). For the purpose of connectivity computation, we considered all pairs of GM contacts, except those that were in the same electrode and within the same ROI.

\subsubsection{Single-trial mean functional connectivity}

We computed the time-varying functional connectivity (FC) between broadband pre-processed signals $(1-700 \mathrm{~Hz})$, which, due to the $1 / f$ filtering effect 
of brain tissue, is dominated by low frequency dynamics. To do so, we used a sliding-window approach with a window length of $200 \mathrm{~ms}$ (410 samples) and a step of 1 sample, thus obtaining a signal with a temporal resolution and precision equivalent to that of the power time courses above $32 \mathrm{~Hz}$ (see section 2.7). Specifically, for each trial and time point, we considered a time window spanning $200 \mathrm{~ms}$ around it, and extracted the FC matrix using the Pearson correlation coefficient between pairs of contacts. Then, we computed the average FC across all selected pairs of contacts (excluding pairs in the same electrode and within the same ROI), thus obtaining a single-trial mean functional connectivity value. When assessing the stimulus-locked global fluctuations, we aligned the $\mathrm{mFC}$ time courses to stimulus presentation, epoched the data from -500 to $1500 \mathrm{~ms}$ around stimulus onset and took the median of the mFC across all trials. For each subject, we visually identified one time window with a significant deflection of the $\mathrm{mFC}$ with respect to the baseline period and tested for significant differences using a Ranksum test across trials on the time average $\mathrm{mFC}$ in the baseline period and in the selected window, with a criterion of $P<0.05$.

On the other hand, when assessing the motor-related global fluctuations (only subject 2), we aligned trials to button press or response timeout end, epoched the data from -500 to $500 \mathrm{~ms}$ from button press or response timeout end, respectively, and computed the median of the mFC across the two sets of trials (recognized, non-recognized), respectively.

\subsubsection{Inter-trial phase-locking value}

To assess the global degree of consistency of phase relationships among recording sites across trials (at each time and frequency point), we defined the mean inter-trial phase-locking value (mITP) as follows. For each contact, phase estimates were obtained using the multitaper method with the same parameters and procedure specified in section 2.7. When assessing the stimulus-locked global fluctuations, phase signals were aligned to stimulus presentation and epoched from -500 to $1500 \mathrm{~ms}$ around stimulus onset. Then, the inter-trial phase-locking value between two contacts $k_{1}, k_{2}$ was computed as the Phase Locking Value (PLV) (Lachaux et al., 2003) across all trials:

$$
\operatorname{ITP}_{k_{1}, k_{2}}(f, t)=\frac{1}{N}\left|\sum_{n=1}^{N} e^{i\left(\phi_{k_{1}, n}(f, t)-\phi_{k_{2}, n}(f, t)\right)}\right|,
$$

where $N$ is the number of trials and $\phi_{k, n}(f, t)$ represents the phase estimate of contact $k$ at frequency $f$ and at time $t$ of the $n$-th trial. ITP ranges from 0 to 1 , where 1 represents equal phase relationships across trials. Finally, the mITP was obtained by averaging the ITP across all selected pairs of contacts (excluding pairs in the same electrode and within the same ROI), 
thus obtaining a single inter-trial time series for all trials.

To study motor-locked global responses (only subject 2), we computed the mITP across the two sets of trials (recognized vs non-recognized), independently. To do so, phase signals were aligned to button press or response timeout end and epoched from -500 to $500 \mathrm{~ms}$ from button press or response timeout end, respectively. Then an mITP time series was derived for each set of trials.

\subsection{Coupling between local activity and global connectivity fluctuations}

To study local-global relationships, we aimed to have one time-varying variable for each recording site to capture local dynamics (derived from the spectrograms) and global time-varying variables capturing fluctuations among recording sites (derived from $\mathrm{mFC}$ and $\mathrm{mITP}$ ). Based on the preliminary visual inspection, we refined our definition of local activity and defined it as high-gamma activity $(64-256 \mathrm{~Hz})$ and we analyzed the stimulus-locked setting (subjects 1 and 2). Hence, for each site, the local variable was defined as the average of its (median) spectrogram within the aforementioned high-frequency range. In contrast, at the global level, we used the median $\mathrm{mFC}$ across all trials. We also defined a second global variable based on the the mITP within the frequency range where it showed the highest stimulusmodulation $(6-16 \mathrm{~Hz})$. To do so, we first z-scored the mITP across the whole epoch (from -500 to 1500 around stimulus presentation) and extracted the average across $6-16 \mathrm{~Hz}$.

To assess the degree of correlation between each contact's local activity and the global connectivity fluctuations measured by the proposed global variables, we used the following procedure. For each recording site, we computed the Spearman correlation coefficient between its local variable and the global variable across the entire epoch. To infer the significance of each estimation, we built the corresponding null distribution using circular shifts of the local variable, which preserved the local and global variables' autocorrelation properties (around $200 \mathrm{~ms}$ by definition) while destroying their temporal alignment. Only medium and large effect size correlations $(r>0.3)$ were kept. In addition, significance level was set to $\alpha=0.05$ and corrected for multiple comparisons using Bonferroni correction (number of contacts).

A similar procedure was used to assess local-global relationships in the motor-locked setting. In this case, local and global variables were aligned to button press, chunked in temporal windows from -500 to $500 \mathrm{~ms}$, and and only obtained for recognized trials. We then assessed the degree of correlation between each recording site's local activity and the global connectivity fluctuations using the procedure described in the previous paragraph for the recognized trials. 


\subsection{Data and code availability}

Due to institutional restrictions, the data that supports the findings of this study can be accessed only with a data sharing agreement. The code used in this study is available from the corresponding author upon request.

\section{Results}

\subsection{Implantation, behavioural task and basic ERP analysis}

We analyzed the intracranial EEG signals from subjects 1 and 2 while they performed a face-recognition task (Fig. 1A). In this task, each trial consisted of a short pre-stimulus baseline period, an image presentation period and a post-stimulus period in which the subject was instructed to respond within a maximum allowed time.

Subject 1 had 16 electrodes implanted, accounting for a total of 175 contacts, among which 46 were selected for further analysis (see Table 1 and Fig. 1B). Electrodes were implanted on the left hemisphere and covered the primary or secondary visual cortex (1 electrode in V1/V2), the supramarginal gyrus (2 electrodes), large portions of the lateral aspect of the temporal lobe (5 electrodes in the anterior and posterior regions of the STG, MTG, and ITG), anterior and posterior parts of the hippocampus (2 electrodes), the cingulate cortex (4 electrodes distributed uniformly from its rostral anterior to its posterior aspect close to the isthmus), and to a lesser extent the frontal lobe (1 electrode in M1, 1 in the lateral aspect of the PMC and 2 in the DLPFC). Subject 1 completed $N=64$ trials that lasted approximately 12 minutes. Around $0.2 \%$ of inspected time windows were marked as corrupt $(7 / 2880)$. However, none of them affected analyzed periods (from $-500 \mathrm{~ms}$ to $1500 \mathrm{~s}$ around stimulus onset) and no trials had to be discarded. ERP in the visual cortex of subject 1 was found to be modulated by the stimulus presentation (Fig. 1C), showing negative peaks of $-20 \mu \mathrm{V}$ at around 100 and $400 \mathrm{~ms}$ after stimulus presentation, with a sudden increase to $30 \mu \mathrm{V}$ at $500 \mathrm{~ms}$ after stimulus presentation.

Subject 2 had 7 electrodes implanted, with a total of 93 contacts, among which 26 were selected for further analysis (see Table 1). Implanted electrodes covered large portions of the frontal lobe (1 electrode in M1, 1 in the medial aspect of the PMC, 2 in the DLPFC and 1 in the VLPFC), the cingulate cortex ( 5 electrodes distributed from its anterior to its posterior aspect), the STG (2 electrodes) and the insula (1 electrode). Subject 2 completed $N=71$ trials that lasted approximately 5 minutes. Around $0.3 \%$ of inspected time windows were marked as corrupt (3/1200). As before, none of them affected analyzed periods (from $-500 \mathrm{~ms}$ to $1500 \mathrm{~s}$ around stimulus onset; from $-500 \mathrm{~ms}$ to $500 \mathrm{~ms}$ around button press or response timeout) and no trials had to be discarded. Among all pictures, 22 were reported as 
recognized, while 49 were reported as non-recognized. In subject 2 , eventrelated potential (ERP) in right-hand M1 (Fig. 1D) displayed significant differences between conditions from -271 to $-210 \mathrm{~ms}$ before button press and from 4 to $191 \mathrm{~ms}$ after button press (Ranksum test at each time point and across conditions, with a criterion of $P<0.05$ for a minimum of 102 consecutive samples, $50 \mathrm{~ms}$ ).

\subsection{Detection of task-driven local activity}

The spectrogram of each signal was obtained using the multitaper method. Power estimates at each frequency were then epoched and expressed as a fraction of the mean power during the baseline period (from -400 to -100 ms from stimulus onset) at each frequency, for each trial separately (see Methods, section 2.7).

Subject 1 had an implantation that largely covered the stimulus processing pathway (Fig. 2A, left panel): V1/V2, ITG, MTG, hippocampus. In this case, we studied stimulus-related activations across all trials and disregarded behavioural responses (see Methods, section 2.2). For every contact, the median of the spectrograms was computed over all face presentation trials. Fig. 2B shows the spectrograms of two contacts of subject 1 monitoring V1/V2 and the middle portion (anterior-posterior axis) of the MTG (mMTG) that displayed significant power activations. In V1/V2, we found that the brain responses to the presentation of the stimulus were characterized by an increase in the theta band immediately after the stimulus onset time $\left(100-600 \mathrm{~ms}, 4-8 \mathrm{~Hz}, P<10^{-5}\right)$ and a high-gamma increase after $300 \mathrm{~ms}\left(300-600 \mathrm{~ms}, 64-256 \mathrm{~Hz}, P<10^{-6}\right)$. In addition, the mMTG exhibited a significant deflection localized in the beta band during the second half of the stimulus period $(16-32 \mathrm{~Hz}, 600-900 \mathrm{~ms}, P<0.001)$, accompanied by a more diffuse activation in the high-gamma range $(64-128 \mathrm{~Hz}$, $500-800 \mathrm{~Hz}, P<0.001)$.

In subject 2 , no ROI related to direct visual stimulus processing was monitored, while various areas related to perceptual decision making and motor report were covered (Fig. 2A, right panel): M1 at the position of the right-hand (used for button pressing), premotor areas, VLPFC and DLPFC. We first performed the stimulus-related analysis. In this setting, we found significant power activations across all trials in the beta band in one contact in the DLPFC and one in M1 during the second half of the stimulus presentation period (16-32 Hz, 500-750, $P<0.01$ for both contacts). Here, we also studied motor-related activations in recognized trials. For every recording site, the median of the spectrograms was computed over each set of trials aligned to button press or response timeout end, respectively (see. Fig. 2C). In this setting, we found significant power activations in the beta band of the the DLPFC before button press (Fig. 2C, 16-32 Hz, from 500 to $250 \mathrm{~ms}$ before button press, $P<0.01$ ). These power activations were, 
nonetheless, non-significant when compared to non-recognized trials. Significant beta power activations were also found in the same time-frequency band in the primary motor cortex $(16-32 \mathrm{~Hz}$, from 500 to $250 \mathrm{~ms}$ before button press, $P<0.01)$. These were also non-significant when compared to non-recognized trials. In addition, M1 displayed high-gamma activations around button press $(64-128 \mathrm{~Hz}$, from $50 \mathrm{~ms}$ before to $200 \mathrm{~ms}$ after button press). Unlike the previous cases, the activations here were significant when compared both to the baseline period $(P<0.01)$ and to the non-recognized trials $(P<0.01)$.

Overall, the detected ROIs at early and late stages of the cortical face recognition and report pathway, i.e. V1/V2 and M1, provided statistical evidence of activations at frequency ranges (Buzsáki et al., 2012) and times (Salinas and Romo, 1998; Van Vugt et al., 2018) that were compatible with local activity. Based on these results, we chose to define the local variables of the local-global analysis within the frequency range $64-256 \mathrm{~Hz}$.

\subsection{Global connectivity effects of stimulus presentation and motor response}

The mean functional connectivity $(\mathrm{mFC})$ and the mean inter-trial phase locking value (mITP) were computed as described in Methods (see section 2.8). The upper panel of Fig. 3 shows the time evolution of the two variables in the stimulus-locked setting (Fig. 3A, mFC; Fig. 3B, mITP). Despite having different implantation schemes, a decrease in the $\mathrm{mFC}$ associated to stimulus presentation was consistently found in both subjects with respect to their pre-stimulus baseline distribution in a temporal window of 300$500 \mathrm{~ms}$. To test significance, a Ranksum test was applied across trials on the mean of the $\mathrm{mFC}$ in the baseline period and the 300-500 ms window $(P<0.05$, Fig. 3A). This decrease in time-resolved functional connectivity (time windows of approximately $200 \mathrm{~ms}$ ) across trials was accompanied by a concurrent decrease in the inter-trial phase locking value (mITP) in the frequency range $6-16 \mathrm{~Hz}$ that lasted $200 \mathrm{~ms}$ (z-score $<3$, Fig. 3B).

To ensure that the reported global effect expressed a genuine connectivity trend of widespread nature we performed two control analysis. First, we inspected the influence of power fluctuations into the mFC decrease by plotting the time-resolved average standard deviation and covariance across all contact pairs in the same period (See Fig. S2) paying special attention at the 300-500 ms window after stimulus onset. The curves illustrate that the decrease was manifested for both subjects in their average covariance (Fig. S2B) while the increase in the average standard deviation only contributed (together with the covariance) to the connectivity decay in subject 2 (Fig. $\mathrm{S} 2 \mathrm{C}$ ). Second, we assessed how the connectivity decay in both $\mathrm{mFC}$ and mITP varied across all contacts (See Fig. S3 and Fig. S4). Indeed, Fig. S3B and Fig. S4B suggest that the period 300-500 ms was affecting the con- 
nectivity strength of a substantial subset of recording contacts (see marked rectangles) while the curves for pre-stimulus and stimulus epochs displayed in Fig. S3C and Fig. S4C confirmed that the decay was a generalized effect across the implantation scheme of both subjects.

With regard to the motor-locked setting, the lower panel of Fig. 3 shows the time evolution of the two global variables for subject 2 . In this setting, trials were aligned to button press or response timeout end, and global variables were computed in a time period from -500 to $500 \mathrm{~ms}$ across recognized $(\mathrm{N}=22)$ and non-recognized ( $\mathrm{N}=49)$ trials, respectively (Fig. 3C, mFC; Fig. $3 \mathrm{D}, \mathrm{mITP})$. In this case, despite the hypothesized presence of statistical effects upon visual inspections, no significant differences with respect to the pre-stimulus baseline or across conditions were found within our dataset.

\subsection{Local-global relationships}

We here investigated the relationship between the local variable of each recording site (average power across the high-gamma range, 64-256 Hz) and the two global connectivity-based variables $(\mathrm{mFC}$, and mITP averaged across the range 6-16 Hz). This relationship was explored in the two proposed settings (stimulus-locked, motor-locked) to find potential local-global couplings associated to stimulus information processing and motor reports (see Methods, section 2.9)

\subsubsection{Stimulus-locked local-global coupling}

Fig. 4 shows the results for the stimulus-locked local-global analysis in subjects 1 (Fig. $4 \mathrm{~A}$ and 4B) and 2 (Fig. $4 \mathrm{C}$ and $4 \mathrm{D}$ ). In particular, the upper left plot in Fig. 4A shows the time evolution of each contact's local variable (average power across the high-gamma range, 64-256 Hz) across the stimulus presentation in subject 1 . Contacts were sorted by the mean highgamma power in the $300-500 \mathrm{~ms}$ time window after stimulus presentation, which defines the task-related activation of each recording site (shown next to each contact's power evolution). The lower plot in Fig. 4A shows the time evolution of the two global variables $(\mathrm{mFC}$, and $\mathrm{mITP}$ averaged across the range $6-16 \mathrm{~Hz}$ ) temporally aligned to the local variables. The results of local-global testing are shown in Fig. 4B for each global variable, separately. To assess the degree of local-global correlation we computed the Spearman correlation coefficient between each local variable and the global variable across the entire epoch. Only medium and large effect-size correlations $(r>$ 0.3 ) were considered. Significance level was set to $\alpha=0.05$ (null distribution built using circular shifts of the local variable) and corrected for multiple comparisons across multiple contacts. In subject 1 , the local increase of activity of high-gamma activity at V1/V2 was found to have a significant correlation of -0.7 with the decrease in the two global variables (see examples 
of downsampled scatter plots for each global variable in S5A). The decrease of $\mathrm{mFC}$ was also significantly correlated with high-gamma activations in other contacts such as mMTG and mITG (middle portions of the MTG and ITG, respectively, $r \approx-0.5$ ), which also ranked high in task-related activations. The fluctuations in mITP across the trials correlated also with other contacts that were less active during the task. A significant negative correlation was found with the PCC. In addition, some contacts that suffered negative deflections in high-gamma activity correlated positively with mITP fluctuations (PMC, posterior portion of the STG, $r \approx 0.5$ ).

In an analogous form, the upper left plot in Fig. $4 \mathrm{C}$ shows the time evolution of the local variable (average power across the high-gamma range, 64-256 Hz) across the stimulus presentation in subject 2. High-gamma activity appears to be far less modulated by the task than in subject 1 (see task-related activation of each recording site next to each contact's power evolution), probably due to an implantation scheme covering regions that were not involved in the initial processing phases of visual stimuli. The lower plot in Fig. 4C shows the time evolution of the two global variables ( $\mathrm{mFC}$, and $\mathrm{mITP}$ averaged across the range 6-16 $\mathrm{Hz}$ ) temporally aligned to the local variables. The results of local-global testing are shown in Fig. 4D for each global variable, separately. Interestingly, although global fluctuations in subject 2 exhibited very similar trends to those observed in subject 1 , no local variable significantly explained the reported fluctuations.

\subsubsection{Motor-locked local-global coupling}

We here tested couplings between the global variables and the local ones when aligned to button press (time period from -500 to $500 \mathrm{~ms}$ from motor report) in subject 2 . Fig. 5 shows the results for motor-locked local-global analysis in recognized trials. The upper left plot in Fig. 5A shows the time evolution of each contact's local variable (average power across the highgamma range, 64-256 Hz) around the motor report in subject 2. Contacts have been sorted by the mean high-gamma power from -100 to $200 \mathrm{~ms}$ from button press, which defines the motor-related activation of each recording site (shown next to each contact's power evolution). The lower plot in Fig. 5A shows the time evolution of the two global variables $(\mathrm{mFC}$, and mITP averaged across the range $6-16 \mathrm{~Hz}$ ) temporally aligned to the local variables. In all figures, pre-stimulus baseline is also shown for comparison although not used for local-global analysis. Local-global correlation was assessed using the same procedure as in the stimulus-locked setting. The results of local-global testing are shown in Fig. 5B for each global variable, separately. Importantly, local increases in activity at M1 were found to be positively correlated $(r=0.5, P<0.05$ corrected for multiple comparisons across contacts; see an exemplary downsampled scatter plot in Fig. S5B left) with $\mathrm{mFC}$, that also exhibited an increase during motor report. Some 
contacts in the DLPFC (see an exemplary downsampled scatter plot in Fig. S5B right) and VLPFC appeared to have significant negative correlations with $\mathrm{mFC}$, some of which showed prominent negative deflections during the motor report period. None of the local variables showed a significant correlation with mITP.

\section{Discussion}

In this study we developed and systematized a methodological pipeline integrating neuroanatomic information, signal processing functions and connectivity measures to statistically infer potential correlations between the local activity and global connectivity fluctuations. This new local-global framework was tested in two epileptic patients with iEEG recordings that performed a face-recognition task.

The crucial point of our framework is the appropriate definition of local and global variables, and their accurate estimation. For the local variables, we used power activations in the high-gamma range $(64-256 \mathrm{~Hz})$ estimated via the continuous multitaper method, which is designed to reduce bias with respect to true spectral content (see Supplementary Information for a comparison and discussion of multitaper and wavelet estimation techniques). The global variables were defined by means of functional connectivity analysis. Crucially, they were based on lower frequency cofluctuations, which are thought to have a more widespread origin (Pesaran et al., 2018), reflecting more global states. Based on these premises, we made use of two independent functions in the low-frequency range to measure the brain sites' coupling consistency across time. On one hand, the mean functional connectivity $(\mathrm{mFC})$ is based on temporal correlations between pairs of broadband signals (which are dominated by low frequencies) and captures temporal cofluctuations across recording sites. On the other hand, the mean intertrial phase-locking value (mITP) relies on instantaneous phase estimation and quantifies the consistency of region phase relationships across different task trials (at each scale). Then, local-global relationships were tested via correlation-based tests, although more sophisticated methods could also be used, as discussed in section 4.4 .

\subsection{Implantation and task analysis}

Despite performing the same task, subjects 1 and 2 had differentiated implantation schemes, which allowed to test our framework with heterogeneous conditions. Overall, ROIs at both ends of the cortical pathway in visual perception and motor report were covered when considering both subjects together. Implantation of subject 1 (Fig. 1B) covered extensively the occipital and temporal lobes, including crucial areas of the cortical pathway in visual face perception and processing (V1/V2, ITG, MTG, hippocampus) 
(Bernstein and Yovel, 2015; Jonas et al., 2016; Wang et al., 2016), while implantation of subject 2 (Fig. 2B) was more extense in the PFC, and motor and premotor areas. Primary results were obtained by aligning trials to stimulus presentation. In addition, we explored modulations aligned to motor-response in recognized trials. Subject 2 was particularly interesting for the purpose of motor-locked analysis, since one contact was located in M1 (left hemisphere), approximately in the region controlling right-hand movement, with which motor reports were made. In this contact, ERPs exhibited a significant deflection when compared to the surrogate condition obtained by using non-recognized trials locked to response timeout end.

\subsection{Task-driven activations}

Following (Rey et al., 2014), we defined certain time-frequency windows of interest (TFOI) in the spectrograms of preselected key recording sites and tested whether activations in those windows were significant with respect to the pre-stimulus presentation or across conditions when aligned to motor response (Fig. 2). Following this approach, in subject 1 we observed significant power increases in V1/V2 in the theta and and high-gamma range during the first half of the stimulus, that were followed by more diffuse activations in the MTG in the beta and high-ramma range during the second half of the stimulus period. In subject 2 we found significant power activations across all trials in the beta band in the DLPFC and M1 during the second half of the stimulus presentation period. When aligined to motor responses, power activations in the high-gamma range emerged around button press in recognized trials for subject 2 .

The above results generalize findings from previous literature related to the face perception pathway found with different recording modalities (Zhen et al., 2013; Rey et al., 2014, 2015; Wang et al., 2016; Grill-Spector et al., 2017; Landi and Freiwald, 2017; Dobs et al., 2019) and help reconstruct additional stages of the visual processing pathway needed to consciously recognize face identities and of the motor planning pathway needed to engage in behaviour. Interestingly, both ends of the cortical pathway (V1/V2 and M1) showed broadband high-frequency activity during stimulus presentation and perceptual report which might reflect the presence of neural populations encoding sensory and motor information in each area, respectively (Buzsáki and Draguhn, 2004). In contrast, intermediate nodes of this pathway such as the MTG area, engaged in processing visual face features, and the DLPFC, engaged in decision planning, exhibited prominent activity in the beta band during the second half of the stimulus presentation. In particular, beta activity in M1 across all trials not concurrent to high-gamma activity might reflect afferent potentials that do not result in local activity.

The encoding of visual stimuli in the visual cortex (Kohn and Smith, 2005; Smith and Kohn, 2008; Graf et al., 2011) and the neural correlates of 
the somatosensory-motor pathway (Salinas and Romo, 1998; Romo et al., 2002; Luna et al., 2005; Thura and Cisek, 2014; Campo et al., 2015) have long been studied in primates. The novelty of our study, however, lies in having been able to generalize some results in human brain recordings. In addition, global fluctuations potentially reflecting cognitive states have been poorly analyzed due to the difficulty of recording simultaneous single neurons during task performance. Intracranial electroencephalography (iEEG) recordings from the human brain provide an opportunity to study such fluctuations at the global scale thanks to their coverage of brain areas so distant such as V1 and M1.

\subsection{Consistency of global patterns independent of implanta- tion scheme}

To characterize global connectivity states during the task, we used two complementary variables. On one hand, the mean functional connectivity ( $\mathrm{mFC})$ was defined as the averaged Pearson correlation across contact pairs in 200 ms time windows, thus capturing cofluctuations of the broadband signals $(1-700 \mathrm{~Hz})$, which are ultimately dominated by low frequencies. On the other hand, the mean inter-trial phase locking value (mITP) was defined to characterize the consistency of phase-relationships among recording sites across trials at each frequency scale. Significant modulations associated to stimulus presentation in this variable were only found in the $6-16 \mathrm{~Hz}$ range. We therefore restricted our analyses to this frequency range for this variable.

Remarkably, despite having differentiated implantation schemes, the connectivity functions consistently showed in both subjects a significant global desynchronization occurring a few hundred milliseconds after stimulus onset (Fig. 3, upper panel), which was shown to be of generalized nature and not specifically biased by power fluctuations (See Figs. S2, S3, S4). This suggests that volume conduction at low frequencies in the monopolar montage might be useful when aiming to capture global connectivity states that are independent of the implantation scheme, as already implied by previous works (Tauste Campo et al., 2018). The decrease in time-resolved $\mathrm{mFC}$ reflects that the stimulus breaks baseline connectivity cofluctuations, owing to a potential specialization or segregation of different subnetworks in processing the incoming information. In addition, the decrease in mITP in the beta band suggests that this processing is stimulus-specific. Note that in our study we used different faces in each trial. Further studies should test whether the trial specificity is maintained when using exactly the same stimulus across trials. When studying motor-locked global effects (subject 2, Fig. 3, lower panel), no clear trend was observed in the mITP, while the $\mathrm{mFC}$ exhibited a transient increase around the response time. These results should be interpreted with care, since the number of recognized trials was as small as 22. Further studies should be performed to assess the consistency 
of reported results with more subjects and with more trials of each kind.

With the results at hand, we hypothesize that the stimulus presentation might initially trigger a specialization of the whole-brain network (reflected in the decrease of $\mathrm{mFC}$ ) to process the incoming stimulus, followed by an increase in connectivity (reflected by the positive deflection in $\mathrm{mFC}$ around response) needed to integrate information and plan an internal response. Yet, the underlying mechanism behind the reported global fluctuations remains unclear. Further studies should assess what other variables (context, other cognitive processes, pre-stimulus cognitive state) might modulate these fluctuations. In particular, more ecological frameworks could be used (Freiwald et al., 2016), for instance using dynamic stimuli, to assess the extent to which the reported deflections depend on the stimulus features.

\subsection{Local-global framework}

The local-global framework was used to test whether the activity of some recording sites could explain this global desynchronization better than others. In particular, we found that the effect was better explained by the local activity of brain areas involved in visual information processing, providing evidence that the global measures might be a novel signature of functional brain activity segregation taking place when a stimulus is processed in a task context. In addition, in the response-locked setting, the increase in mFC was better explained by the local activity of brain areas in the motor cortex.

Here, we propose a first study to quantitatively asses local-global relationships in a task-related context. This study should pave the way for more sophisticated methods that can assess the role of each node when considering the whole network together. Generalized Linear Models (GLMs), for instance, could serve this purpose. However, a difficulty in pursuing this approach lies in having insufficient statistical power, specially in short trials with a large number of recording sites. In addition, latent non-observed variables should be considered to control for key hubs in the network that are not monitored but might coordinate global fluctuations. As previously mentioned, such improved models should take into account the modulation of the local-global coupling by other contextual variables or previous cognitive states. A further improvement of the methodology should assess causality in local-global brain effects, for instance by identifying particular global brain states that trigger local activity at certain nodes. This could be done by means of directionality measures such as Granger causality or directed information (Tauste Campo, 2020).

Another issue to take into consideration is the different time scales of local and global fluctuations. Physiologically meaningful local events such as HFOs or neuronal encoding can occur on the order of milliseconds (Romo et al., 2002; Arnulfo et al., 2015). At the same time, some studies have found 
global fluctuations with characteristic timescales of tens of seconds. In particular, resting state networks (RSN) below $0.1 \mathrm{~Hz}$ have been identified under the resting state condition both with fMRI and with time-resolved MEG (De Pasquale et al., 2010; Brookes et al., 2011; Hipp et al., 2012; Buckner et al., 2013). Other studies based on computational modelling or statistical inference have found global network states with a lifetime of around $200 \mathrm{~ms}$ (Buckner et al., 2013; Deco et al., 2019). This growing evidence suggests that different brain phenomena can be characterized by diverse spatial scales and evolve over the course of different temporal scales (Northoff et al., 2020; Vila-Vidal et al., 2020). Experimental designs and models linking activity at different spatial scales will have to face this phenomenon. Long resting-state iEEG recordings, for instance, could be used to test this hypothesis based on local intrinsic fast activations linked to slower-changing global states.

\subsection{Study limitations}

The local-global framework developed here has been used to explain the variability across time of trial-average global variables by means of trial-average local variables. By taking averages across trials, this approach disregards another source of variability arising from inter-trial differences. Although out of the scope of this study, our framework could be adapted to regress the variablity of global variables across trials at each time point using the local variables. Note that this could be done with $\mathrm{mFC}$, but not with mITP, since trial resolution is lost upon computation of this variable. A possible alternative would be to use measures than can be computed on a trial-by-trial basis using time-frequency windows, such as the PLV or other phase-based measures. However, time autocorrelation in low frequencies can suppose a major drawback in pursuing this approach, specially in short trials where slow fluctuations cannot be captured. Although this study has a methodological nature, some of the results reported here should be validated with a larger number of subjects. In particular, patients should be chosen carefully according to their implantation schemes to better cover the visual-motor pathway in the aim to refine and better understand the bidirectional effect between local activity and global fluctuations during this type of task.

\section{Acknowledgments}

We thank all subjects for their participation in the study. MVV was supported by a fellowship from "la Caixa" Foundation, Spain (ID 100010434, fellowship code LCF/BQ/DE17/11600022). MVV and ATC were supported by the Bial Foundation grant 106/18. GD and ATC were supported by the project "Clúster Emergent del Cervell Humà" (CECH, ref. 001-P-001682), within the framework of the European Research Development Fund Operational Program of Catalonia 2014-2020. GD was supported by a Spanish na- 
tional research project (ref. PID2019-105772GB-I00 MCIU AEI) funded by the Spanish Ministry of Science, Innovation and Universities (MCIU), State Research Agency (AEI); HBP SGA3 Human Brain Project Specific Grant Agreement 3 (grant agreement no. 945539), funded by the EU H2020 FET Flagship programme; SGR Research Support Group support (ref. 2017 SGR 1545), funded by the Catalan Agency for Management of University and Research Grants (AGAUR); Neurotwin Digital twins for model-driven noninvasive electrical brain stimulation (grant agreement ID: 101017716) funded by the EU H2020 FET Proactive programme; euSNN European School of Network Neuroscience (grant agreement ID: 860563) funded by the EU H2020 MSCA-ITN Innovative Training Networks; Brain-Connects: Brain Connectivity during Stroke Recovery and Rehabilitation (id. 201725.33) funded by the Fundacio La Marato TV3; Corticity, FLAG-ERA JTC 2017, (ref. PCI2018-092891) funded by the Spanish Ministry of Science, Innovation and Universities (MCIU), State Research Agency (AEI). 


\begin{tabular}{|c|c|c|c|c|c|c|c|c|c|}
\hline Subject & Sex & $\begin{array}{l}\text { Age } \\
\text { (years) }\end{array}$ & $\begin{array}{l}\text { Epilepsy } \\
\text { onset } \\
\text { (years) }\end{array}$ & $\begin{array}{l}\text { Suspected } \\
\text { epileptic } \\
\text { focus }\end{array}$ & $\begin{array}{l}\text { Hand } \\
\text { laterality }\end{array}$ & Implantation & $\begin{array}{l}\text { Number of } \\
\text { electrodes } \\
\text { (right/left) }\end{array}$ & $\begin{array}{l}\text { Number of } \\
\text { contacts } \\
\text { (right/left) }\end{array}$ & $\begin{array}{l}\text { Number of } \\
\text { contacts } \\
\text { included } \\
\text { in analysis }\end{array}$ \\
\hline 1 & F & 51 & 27 & $\begin{array}{l}\text { Right lateral } \\
\text { frontal / insula } \\
\text { (FCD) }\end{array}$ & Right & Right & $16 / 0$ & $175 / 0$ & 46 \\
\hline 2 & M & 49 & 15 & $\begin{array}{l}\text { Left dorso- } \\
\text { medial frontal }\end{array}$ & Right & Left & $0 / 7$ & $0 / 93$ & 26 \\
\hline
\end{tabular}

Table 1: Demographic data. F: female; M: male; FCD: focal cortical dysplasia

\begin{tabular}{l|cc|cc}
\hline ROI & \multicolumn{2}{|c|}{ P1 } & \multicolumn{2}{|c}{ P2 } \\
\hline & \#Electrodes & \#Contacts & \#Electrodes & \#Contacts \\
\hline Rostral anterior cingulate & 1 & 1 & 2 & 3 \\
Caudal anterior cingulate & 1 & 2 & 1 & 1 \\
Posterior cingulate & 1 & 2 & 1 & 2 \\
Isthmus cingulate & 1 & 2 & 1 & 1 \\
Insula & 0 & 0 & 1 & 1 \\
Cuneus & 1 & 2 & 0 & 0 \\
Supramarginal & 2 & 7 & 0 & 0 \\
Inferior temporal & 1 & 1 & 0 & 0 \\
Middle temporal & 2 & 6 & 0 & 0 \\
Superior temporal & 2 & 8 & 2 & 6 \\
Superior frontal & 0 & 0 & 1 & 1 \\
Precentral & 1 & 3 & 1 & 3 \\
Pars triangularis & 0 & 0 & 1 & 3 \\
Caudal middle frontal & 1 & 3 & 0 & 0 \\
Rostral middle frontal & 2 & 5 & 2 & 5 \\
Hippocampus & 2 & 4 & 0 & 0 \\
\hline Total & 18 & 46 & 13 & 26 \\
\hline
\end{tabular}

Table 2: Implantation scheme. Regions of interest monitored in each patient are expressed in terms of the Desikan-Killiany atlas with an extra ROI for the hippocampus. 
bioRxiv preprint doi: https://doi.org/10.1101/2021.06.25.449912; this version posted August 14, 2021. The copyright holder for this preprint (which was not certified by peer review) is the author/funder, who has granted bioRxiv a license to display the preprint in perpetuity. It is made available under aCC-BY-NC-ND 4.0 International license.

A

One trial

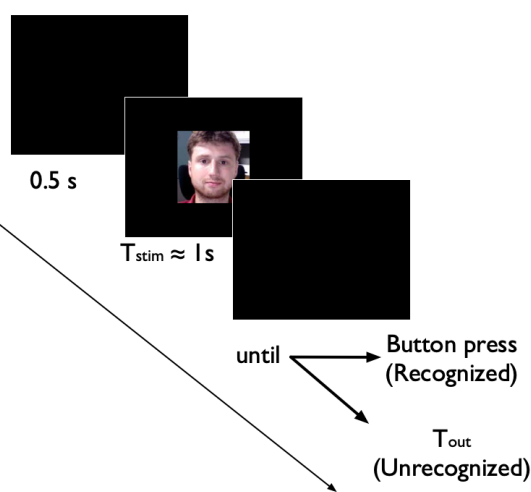

C

C

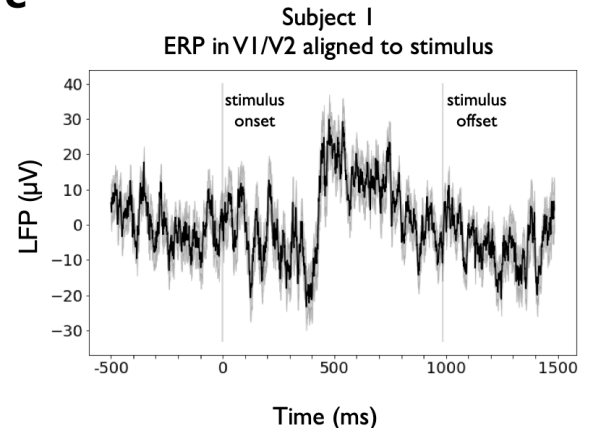

B
Subject I

Contact in VI/V2 highlighted


D

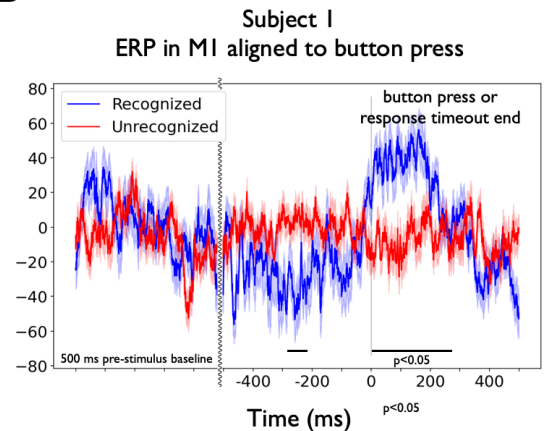

Figure 1: Experimental protocol, recording locations and neural responses.(Continues in next page) 
Figure 1: (from previous page) Experimental protocol, recording locations and neural responses. (A) Schematic representation of the behavioural task. Each trial consists of a pre-stimulus blank screen lasting $0.5 \mathrm{~ms}$, a face image that remains present for approximately $1 \mathrm{~s}$ and a blank screen, where the subject has to report whether they recognized the person by pressing a trigger button. Alternatively, the trial ends after a maximum response timeout $T_{\text {out }}$. (B) Subject 1 post-implantation MRI brain scans showing different electrode trajectories in sagittal, coronal and horizontal planes (from top left to bottom right) and $3 \mathrm{D}$ brain reconstruction (top right). The red circles highlight the trajectory of the electrode pointing towards the primary and secondary visual areas. (C) Eventrelated potential (ERP) in V1/V2 (deepest electrode contact of the trajectory highlighted in (B)) aligned to stimulus presentation (median $\pm \mathrm{SEM}$ across $\mathrm{N}=64$ trials). Signals were baseline-corrected on a trial-by-trial basis (baseline from $500 \mathrm{~ms}$ to $0 \mathrm{~ms}$ before stimulus presentation) before averaging. Vertical dark lines indicate the stimulus onset and offset times, respectively. (D) Event-related potential (ERP) in M1 (right-hand region) during recognized (button press with left hand, blue, median \pm SEM across $N=21$ trials) and non-recognized (timeout end, red, median \pm SEM across $\mathrm{N}=49$ trials) trials aligned to button press or response timeout end, respectively (vertical dark line). Signals were baseline-corrected on a trial-by-trial basis (baseline from $500 \mathrm{~ms}$ to $0 \mathrm{~ms}$ before stimulus presentation) before averaging. Pre-stimulus baseline is also shown for comparison. Curvy line marks time discontinuity. In this case, we tested differences in the signals between both conditions. Black bars indicate time periods with significant differences between conditions (Ranksum test at each time point and across conditions, with a criterion of $P<0.05$ for a minimum of 102 consecutive samples, $50 \mathrm{~ms}$ )

A

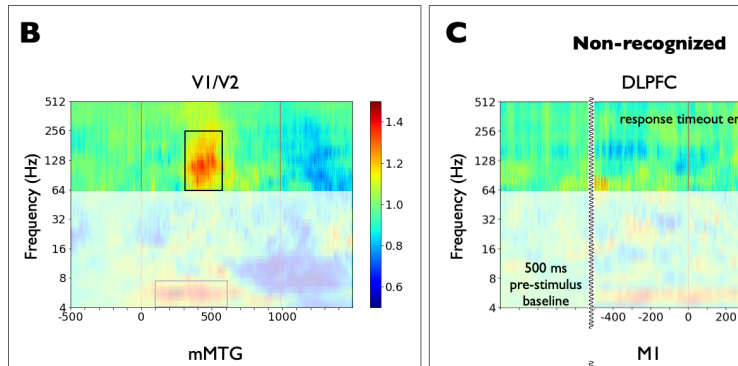

DLPFC



MI

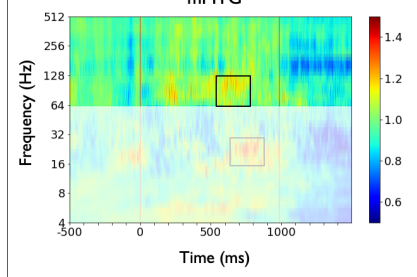

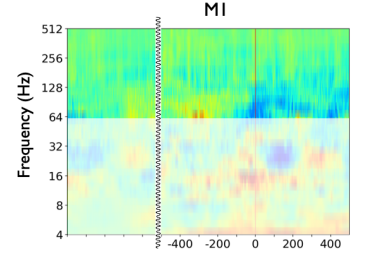

Time (ms)

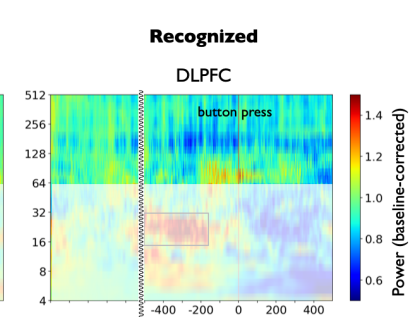

MI

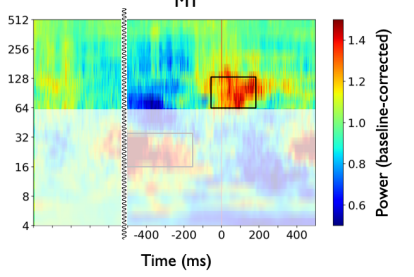

Figure 2: Spectral activations in different task-relevant ROIs for each subject. 
Figure 2: (from previous page) Spectral activations in different task-relevant ROIs for each subject. (A) 3D brain reconstructions showing the electrode trajectories for subjects 1 and 2. Black dots represent electrode trajectory entry points. Red dots highlight electrodes for which spectrograms are shown in (B) and (C). (B) Median across all trials of the baseline-corrected spectrograms aligned to stimulus presentation $(0 \mathrm{~ms})$ and obtained from two different key recording sites in the visual stimulus processing pathway of subject 1. The time-frequency windows of interest (TFOIs) used for statistical comparisons are marked with black rectangles. There is an early power increase in the theta band $(P<$ $10^{-5}$ ) in the visual cortex (V1/V2, same contact as in Fig. 1C) followed by high-gamma discharges $\left(P<10^{-6}\right)$. In the mMTG (middle portion in the anterior-posterior axis of the middle temporal gyrus) we found power activations during the second half of the stimulus presentation both in the beta $(P<0.001)$ and the high-gamma range $(P<0.001)$. Vertical red lines indicate the stimulus onset and offset times, respectively. (C) Median baseline-corrected spectrogram of the single-trial LFP power across recognized and nonrecognized trials aligned to button press or response timeout end ( $0 \mathrm{~ms}$, red vertical line), respectively, obtained from two different areas related to perceptual decision making and motor report. Power increases in the beta band until $250 \mathrm{~ms}$ before button press were found to be significant both in DLPFC (dorsolateral prefrontal cortex) and M1 (same contact as in Fig. 1D) with respect to baseline distribution $(P<0.01)$, but not significant with respect to non-recognized trials. A significant increase in high-gamma power was found in M1 around button press both with respect to baseline $(P<0.01)$ and to the non-recognized trials $(P<0.01)$. Pre-stimulus baseline is also shown for comparison. Curvy lines mark a discontinuity in time. 
bioRxiv preprint doi: https://doi.org/10.1101/2021.06.25.449912; this version posted August 14, 2021. The copyright holder for this preprint (which was not certified by peer review) is the author/funder, who has granted bioRxiv a license to display the preprint in perpetuity. It is made available under aCC-BY-NC-ND 4.0 International license.
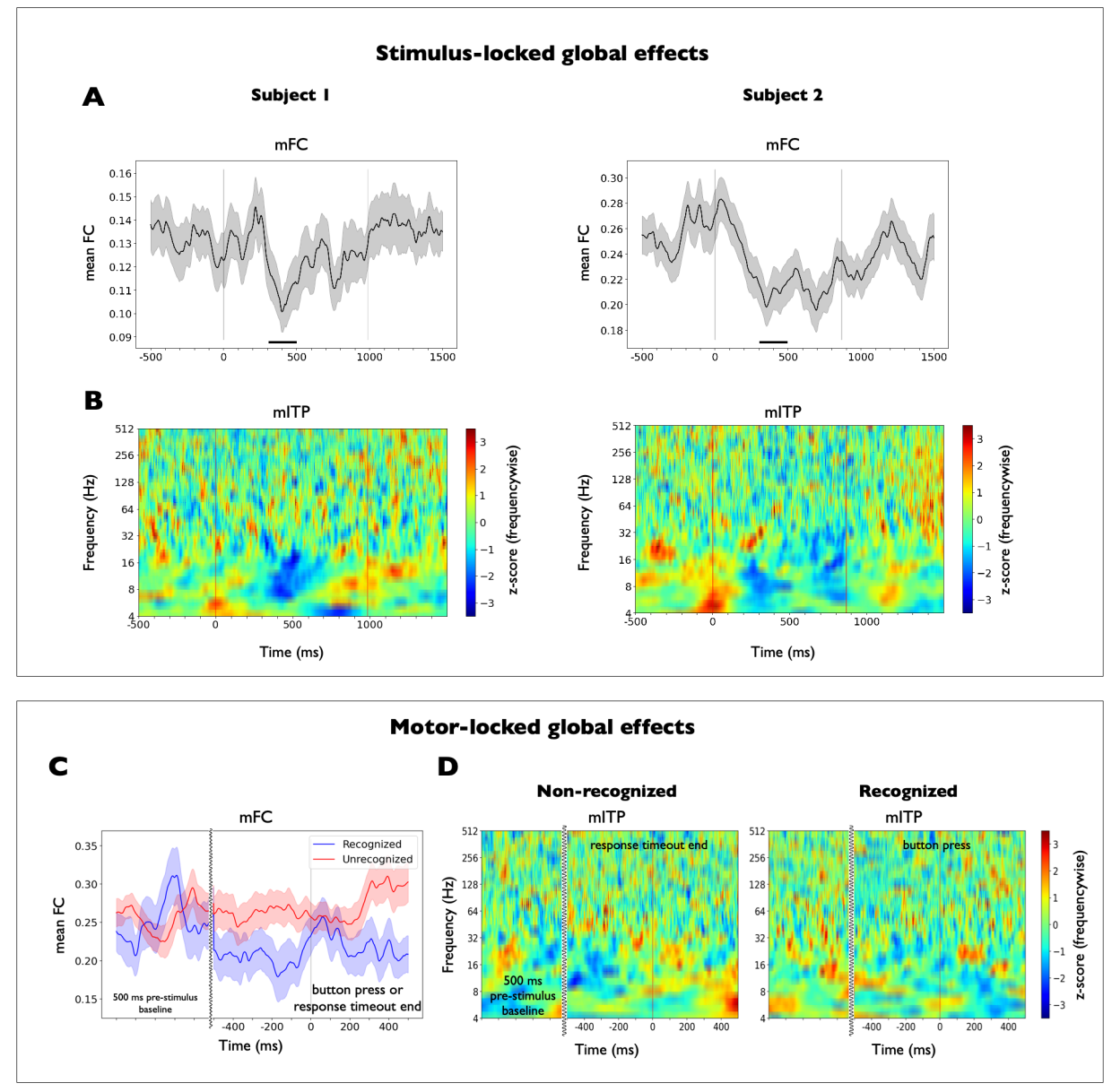

Figure 3: Stimulus- and motor-related global effects. 
Figure 3: (from previous page) Stimulus- and motor-related global effects. The upper panel shows the temporal evolution of the global variables aligned to stimulus presentation across all trials in subjects 1 and 2. (A) Time course of the mean functional connectivity (mFC). Median \pm SEM across all trials aligned to stimulus onset. See Methods, section 2.8 , for details. Vertical dark lines indicate the stimulus onset and offset times, respectively. A significant decrease in the $\mathrm{mFC}$ associated to stimulus presentation was found in the two subjects with respect to their pre-stimulus mean $\mathrm{mFC}$ value $(300-500 \mathrm{~ms}$, marked with black bars, Ranksum test applied across trials on the mean of the $\mathrm{mFC}$ in the baseline period and the 300-500 ms window, $P<0.05$ ). (B) Time-frequency plots of the mean inter-trial phase locking value (mITP) aligned to stimulus presentation. See Method, section 2.8, for details. The plots have been z-scored at each frequency scale. Vertical red lines indicate stimulus onset and offset times, respectively. A significant phase-decoupling was consistently observed across trials in the frequency range 6-16 Hz around $300 \mathrm{~ms}$ after stimulus presentation in the two subjects $(\mathrm{z}$-score $<3)$. This inter-trial decoupling lasted around $200 \mathrm{~ms}$. The lower panel shows the evolution of the global variables aligned to the behavioural response across the corresponding set of trials in subject 2. (C) Time course of the mean functional connectivity $(\mathrm{mFC})$ for subject 2 in recognized $(\mathrm{N}=22)$ and non-recognized $(\mathrm{N}=49)$ trials. Median $\pm \mathrm{SEM}$ across each set of trials aligned to button press or response timeout end (marked with a vertical dark line), respectively. No significant difference was found. Pre-stimulus baseline is also shown for comparison. Curvy lines mark a discontinuity in time. (D) Time-frequency plots of the mean inter-trial phase locking value $(\mathrm{mITP})$ for subject 2 in recognized $(\mathrm{N}=22)$ and non-recognized $(\mathrm{N}=49)$ trials, aligned to button press or response timeout end, respectively. The plots have been z-scored at each frequency scale. Vertical red lines indicate button press or response timeout end, respectively. Pre-stimulus baseline is also shown for comparison. Curvy lines mark a discontinuity in time. No clear difference could be found between both conditions. 
bioRxiv preprint doi: https://doi.org/10.1101/2021.06.25.449912; this version posted August 14, 2021. The copyright holder for this preprint (which was not certified by peer review) is the author/funder, who has granted bioRxiv a license to display the preprint in perpetuity. It is made available under aCC-BY-NC-ND 4.0 International license.

A

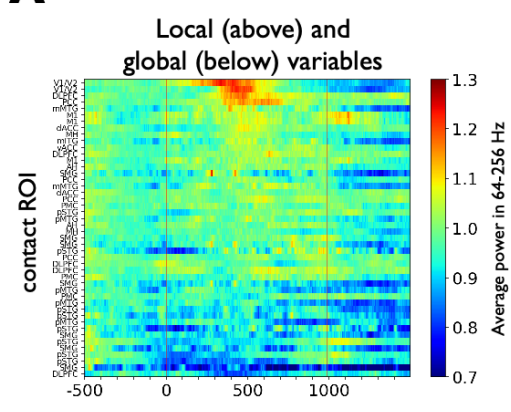

$\dot{<}$

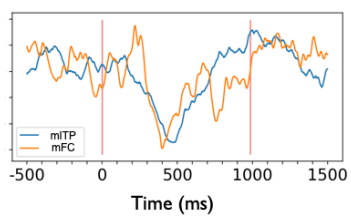

Subject 2

C



B

Task-related

activation
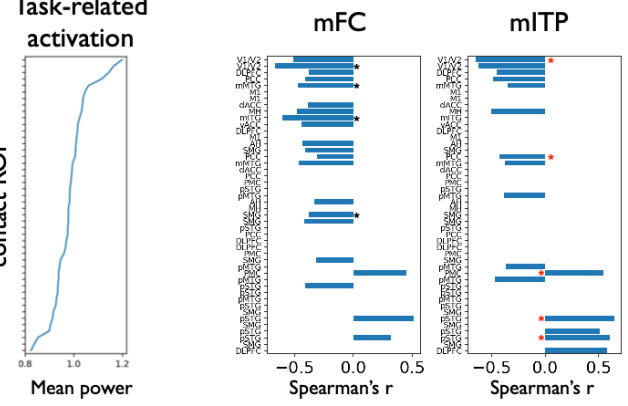

D

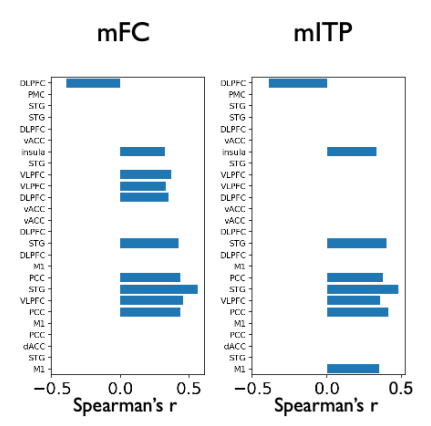

Figure 4: Stimulus-related local-global coupling. 
Figure 4: (from previous page) Stimulus-locked local-global coupling for subjects 1 (upper panel) and 2 (lower pannel). (A) Local and global variables for subject 1. (Upper) Local variable for each brain recording site in subject 1 (median spectrogram power across all trials, averaged across the high-gamma range, 64-256 Hz, see Methods, section 2.9). Vertical red lines indicate the stimulus onset and offset times, respectively. Contacts have been sorted by the mean high-gamma power in the 300-500 ms time window after stimulus presentation, which defines the task-related activation of each contact (shown next to each contact's power evolution). (Lower) Global variables: mFC, and mITP averaged within the frequency range $6-16 \mathrm{~Hz}$. Global variables have been rescaled for the purpose of comparison (shown in arbitrary units, A. U.). Vertical red lines indicate the stimulus onset and offset times, respectively. (B) Spearman correlation coefficient between each local variable and each global variable in subject 1 . Correlations have been thresholded at medium-size effects $(r>0.3)$. In addition, significance was tested using a surrogate distribution via circular shifts, with a significance criterion of $\alpha=0.05$ and corrected for multiple comparisons for the number of contacts. Local increase in activity at V1/V2 appears to have a significant correlation of -0.7 with the decrease in the two global variables. In addition, The decrease in $\mathrm{mFC}$ seems to have a significant correlation with local activity in relevant contacts such as mMTG and mITG (middle portions of the MTG and ITG, respectively). The local activity at PCC appears to be negatively correlated with mITP. (C) Local and global variables for subject 2. Analogous to (A) (D) Spearman correlation coefficient between each local variable and each global variable for subject 2. Analogous to (B). However, in this case, none of the local variables showed a significant correlation with the global variables. 


\section{Recognized trials}
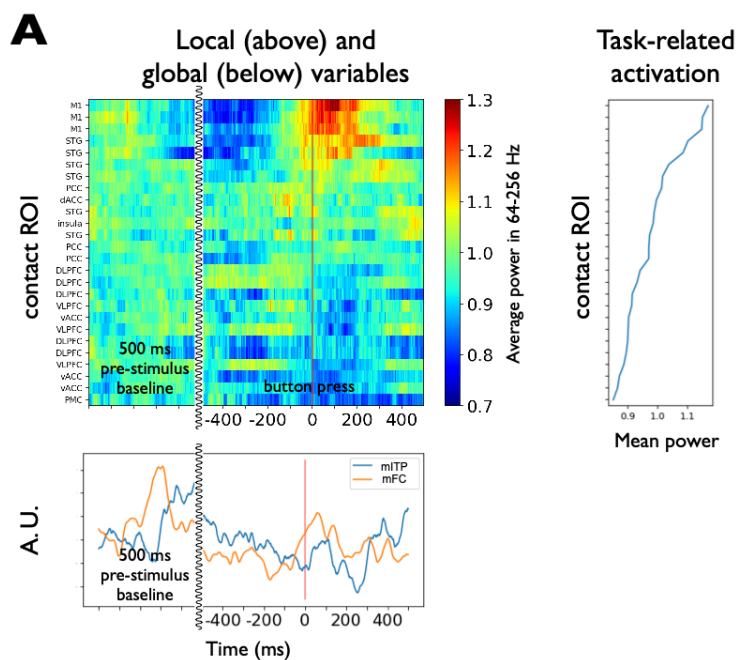

B

$\mathrm{mFC}$



Figure 5: Motor-related local-global coupling for subjects 2 in recognized trials. (A) Local and global variables for recognized trials. (Upper) Local variable for each brain recording site in subject 2 aligned to motor report (median spectrogram power across recognized trials aligned to button press, averaged across the high-gamma range, 64-256 $\mathrm{Hz}$, see Methods, section 2.9). The vertical red line indicates button press. Contacts have been sorted by the mean high-gamma power in a time window around button press (from -100 to $200 \mathrm{~ms}$ ), which defines the motor-related activation of each contact (shown next to each contact's power evolution). (Lower) Global variables: $\mathrm{mFC}$, and mITP averaged within the frequency range 6-16 Hz. Global variables have been rescaled for the purpose of comparison (shown in arbitrary units, A. U.). The vertical red line indicates button press. Pre-stimulus baseline is also shown for comparison (not used for correlations). Curvy lines mark a discontinuity in time. No clear difference was found between the two conditions. (B) Spearman correlation coefficient between each local variable and each global variable in the recognized trials during the time period of -500 to $500 \mathrm{~ms}$ around button press. Correlations have been thresholded at medium-size effects $(r>0.3)$. Significance was tested using a surrogate distribution via circular shifts, with a significance criterion of $\alpha=0.05$ and corrected for multiple comparisons for the number of contacts. Local increase in activity at M1 has a significant positive correlation of 0.5 with $\mathrm{mFC}$. Some contacts in DLPFC and VLPFC appear to have singificant negative correlations with $\mathrm{mFC}$, but their power activations in the high-gamma range are much lower than those of M1. None of the local variables showed a significant correlation with mITP. 


\section{References}

Arnulfo, G., Hirvonen, J., Nobili, L., Palva, S., and Palva, J. M. (2015). Phase and amplitude correlations in resting-state activity in human stereotactical EEG recordings. NeuroImage, 112:114-127.

Axmacher, N., Schmitz, D. P., Wagner, T., Elger, C. E., and Fell, J. (2008). Interactions between medial temporal lobe, prefrontal cortex, and inferior temporal regions during visual working memory: A combined intracranial EEG and functional magnetic resonance imaging study. Journal of Neuroscience, 28(29):7304-7312.

Bassett, D. S., Wymbs, N. F., Porter, M. A., Mucha, P. J., Carlson, J. M., and Grafton, S. T. (2011). Dynamic reconfiguration of human brain networks during learning. Proceedings of the National Academy of Sciences of the United States of America, 108(18):7641-7646.

Bernstein, M. and Yovel, G. (2015). Two neural pathways of face processing: A critical evaluation of current models. Neuroscience and Biobehavioral Reviews, 55:536-546.

Bressler, S. L. and Menon, V. (2010). Large-scale brain networks in cognition: emerging methods and principles. Trends in Cognitive Sciences, 14(6):277-290.

Brookes, M. J., Woolrich, M., Luckhoo, H., Price, D., Hale, J. R., Stephenson, M. C., Barnes, G. R., Smith, S. M., and Morris, P. G. (2011). Investigating the electrophysiological basis of resting state networks using magnetoencephalography. Proceedings of the National Academy of Sciences of the United States of America, 108(40):16783-16788.

Brunel, N. and Wang, X. J. (2001). Effects of neuromodulation in a cortical network model of object working memory dominated by recurrent inhibition. Journal of Computational Neuroscience, 11(1):63-85.

Buckner, R. L., Krienen, F. M., and Yeo, B. T. (2013). Opportunities and limitations of intrinsic functional connectivity MRI. Nature Neuroscience, 16(7):832-837.

Buzsáki, G., Anastassiou, C. A., and Koch, C. (2012). The origin of extracellular fields and currents-EEG, ECoG, LFP and spikes. Nature Reviews Neuroscience, 13(6):407-420.

Buzsáki, G. and Draguhn, A. (2004). Neuronal olscillations in cortical networks. Science, 304(5679):1926-1929.

Campo, A. T., Martinez-Garcia, M., Nácher, V., Luna, R., Romo, R., and Deco, G. (2015). Task-driven intra- and interarea communications in 
primate cerebral cortex. Proceedings of the National Academy of Sciences of the United States of America, 112(15):4761-4766.

Cardinale, F., Cossu, M., Castana, L., Casaceli, G., Schiariti, M. P., Miserocchi, A., Fuschillo, D., Moscato, A., Caborni, C., Arnulfo, G., and Lo Russo, G. (2013). Stereoelectroencephalography: Surgical methodology, safety, and stereotactic application accuracy in 500 procedures. Neurosurgery, 72(3):353-366.

Cohen, M. X. (2019). A better way to define and describe Morlet wavelets for time-frequency analysis. NeuroImage, 199:81-86.

Cruzat, J., Deco, G., Tauste-Campo, A., Principe, A., Costa, A., Kringelbach, M. L., and Rocamora, R. (2018). The dynamics of human cognition: Increasing global integration coupled with decreasing segregation found using iEEG. NeuroImage, 172:492-505.

De Pasquale, F., Della Penna, S., Snyder, A. Z., Lewis, C., Mantini, D., Marzetti, L., Belardinelli, P., Ciancetta, L., Pizzella, V., Romani, G. L., and Corbetta, M. (2010). Temporal dynamics of spontaneous MEG activity in brain networks. Proceedings of the National Academy of Sciences of the United States of America, 107(13):6040-6045.

Deco, G., Cruzat, J., and Kringelbach, M. L. (2019). Brain songs framework used for discovering the relevant timescale of the human brain. Nature Communications, 10(1):1-13.

Deco, G. and Rolls, E. T. (2005). Attention, short-term memory, and action selection: A unifying theory. Progress in Neurobiology, 76(4):236-256.

Deco, G., Tononi, G., Boly, M., and Kringelbach, M. L. (2015). Rethinking segregation and integration: Contributions of whole-brain modelling. Nature Reviews Neuroscience, 16(7):430-439.

Desikan, R. S., Ségonne, F., Fischl, B., Quinn, B. T., Dickerson, B. C., Blacker, D., Buckner, R. L., Dale, A. M., Maguire, R. P., Hyman, B. T., Albert, M. S., and Killiany, R. J. (2006). An automated labeling system for subdividing the human cerebral cortex on MRI scans into gyral based regions of interest. NeuroImage, 31(3):968-980.

Dobs, K., Isik, L., Pantazis, D., and Kanwisher, N. (2019). How face perception unfolds over time. Nature Communications, 10(1):1-10.

Engel, A. K., Moll, C. K., Fried, I., and Ojemann, G. A. (2005). Invasive recordings from the human brain: Clinical insights and beyond. Nature Reviews Neuroscience, 6(1):35-47. 
Freiwald, W., Duchaine, B., and Yovel, G. (2016). Face Processing Systems: From Neurons to Real-World Social Perception. Annual Review of Neuroscience, 39:325-346.

Gabor, D. (1946). Theory of communication. Part 1: The analysis of information. Journal of the Institution of Electrical Engineers - Part III: Radio and Communication Engineering, 93(26):429-441.

Graf, A. B., Kohn, A., Jazayeri, M., and Movshon, J. A. (2011). Decoding the activity of neuronal populations in macaque primary visual cortex. In Nature Neuroscience, volume 14, pages 239-247. Nature Publishing Group.

Grill-Spector, K., Weiner, K. S., Kay, K., and Gomez, J. (2017). The Functional Neuroanatomy of Human Face Perception. Annual Review of Vision Science, 3:167-196.

Hipp, J. F., Hawellek, D. J., Corbetta, M., Siegel, M., and Engel, A. K. (2012). Large-scale cortical correlation structure of spontaneous oscillatory activity. Nature Neuroscience, 15(6):884-890.

Hubel, D. H. and Wiesel, T. N. (1959). Receptive fields of single neurones in the cat's striate cortex. The Journal of Physiology, 148(3):574-591.

Jonas, J., Jacques, C., Liu-Shuang, J., Brissart, H., Colnat-Coulbois, S., Maillard, L., and Rossion, B. (2016). A face-selective ventral occipitotemporal map of the human brain with intracerebral potentials. Proceedings of the National Academy of Sciences of the United States of America, 113(28):E4088-E4097.

Kahana, M. J. (2006). The cognitive correlates of human brain oscillations. Journal of Neuroscience, 26(6):1669-1672.

Kahane, P., Minotti, L., Hoffmann, D., Lachaux, J.-P., and Ryvlin, P. (2003). Invasive EEG in the definition of the seizure onset zone: depth electrodes. Handbook of Clinical Neurophysiology, 3:109-133.

Kohn, A. and Smith, M. A. (2005). Stimulus dependence of neuronal correlation in primary visual cortex of the macaque. Journal of Neuroscience, 25(14):3661-3673.

Lachaux, J.-P., Axmacher, N., Mormann, F., Halgren, E., and Crone, N. E. (2012). High-frequency neural activity and human cognition: Past, present and possible future of intracranial EEG research. Progress in Neurobiology, 98(3):279-301.

Lachaux, J. P., Rudrauf, D., and Kahane, P. (2003). Intracranial EEG and human brain mapping. In Journal of Physiology Paris, volume 97, pages 613-628. Elsevier. 
Landi, S. M. and Freiwald, W. A. (2017). Two areas for familiar face recognition in the primate brain. Science, 357(6351):591-595.

Lopez-Persem, A., Bastin, J., Petton, M., Abitbol, R., Lehongre, K., Adam, C., Navarro, V., Rheims, S., Kahane, P., Domenech, P., and Pessiglione, M. (2020). Four core properties of the human brain valuation system demonstrated in intracranial signals. Nature Neuroscience, 23(5):664-675.

Luna, R., Hernández, A., Brody, C. D., and Romo, R. (2005). Neural codes for perceptual discrimination in primary somatosensory cortex. Nature Neuroscience, 8(9):1210-1219.

Mitra, P. P. and Pesaran, B. (1999). Analysis of dynamic brain imaging data. Biophysical Journal, 76(2):691-708.

Morlet, J., Arens, G., Fourgeau, E., and Giard, D. (1982). Wave propagation and sampling theory - Part II. Sampling theory and complex waves. Geophysics, 47(2):222-236.

Munari, C. and Bancaud, J. (1985). The role of stereoelectroencephalography (SEEG) in the evaluation of partial epileptic seizures. In Porter, R. J. and Morselli, P., editors, The Epilepsies, pages 267-306. Butterworths, London.

Narizzano, M., Arnulfo, G., Ricci, S., Toselli, B., Tisdall, M., Canessa, A., Fato, M. M., and Cardinale, F. (2017). SEEG assistant: A 3DSlicer extension to support epilepsy surgery. BMC Bioinformatics, 18(1):124.

Northoff, G., Wainio-Theberge, S., and Evers, K. (2020). Is temporo-spatial dynamics the "common currency" of brain and mind? In Quest of "Spatiotemporal Neuroscience". Physics of Life Reviews, 33:34-54.

Palva, S., Monto, S., and Palva, J. M. (2010). Graph properties of synchronized cortical networks during visual working memory maintenance. NeuroImage, 49(4):3257-3268.

Pesaran, B., Vinck, M., Einevoll, G. T., Sirota, A., Fries, P., Siegel, M., Truccolo, W., Schroeder, C. E., and Srinivasan, R. (2018). Investigating large-scale brain dynamics using field potential recordings: Analysis and interpretation. Nature Neuroscience, 21(7):903-919.

Rey, H. G., Ahmadi, M., and Quian Quiroga, R. (2015). Single trial analysis of field potentials in perception, learning and memory. Current Opinion in Neurobiology, 31:148-155.

Rey, H. G., Fried, I., and Quian Quiroga, R. (2014). Timing of singleneuron and local field potential responses in the human medial temporal lobe. Current Biology, 24(3):299-304. 
Romo, R., Hernández, A., Zainos, A., Lemus, L., and Brody, C. D. (2002). Neuronal correlates of decision-making in secondary somatosensory cortex. Nature Neuroscience, 5(11):1217-1225.

Russell, B. and Han, J. (2016). Jean Morlet and the Continuous Wavelet Transform. Morlet and the Continuous Wavelet Transform CREWES Research Report, 28(1946):1-15.

Salinas, E. and Romo, R. (1998). Conversion of sensory signals into motor commands in primary motor cortex. Journal of Neuroscience, 18(1):499511.

Siebenhühner, F., Wang, S. H., Arnulfo, G., Lampinen, A., Nobili, L., Palva, J. M., and Palva, S. (2020). Genuine cross-frequency coupling networks in human resting-state electrophysiological recordings. PLoS Biology, 18(5):e3000685.

Slepian, D. and Pollak, H. O. (1961). Prolate Spheroidal Wave Functions, Fourier Analysis and Uncertainty - I. Bell System Technical Journal, 40(1):43-63.

Smith, M. A. and Kohn, A. (2008). Spatial and temporal scales of neuronal correlation in primary visual cortex. Journal of Neuroscience, 28(48):12591-12603.

Sporns, O., Tononi, G., and Kötter, R. (2005). The human connectome: A structural description of the human brain. PLoS Computational Biology, 1(4):0245-0251.

Tauste Campo, A. (2020). Inferring neural information flow from spiking data. Computational and Structural Biotechnology Journal, 18:2699-2708.

Tauste Campo, A., Principe, A., Ley, M., Rocamora, R., and Deco, G. (2018). Degenerate time-dependent network dynamics anticipate seizures in human epileptic brain. PLoS Biology, 16(4):e2002580.

Thomson, D. J. (1982). Spectrum Estimation and Harmonic Analysis. Proceedings of the IEEE, 70(9):1055-1096.

Thura, D. and Cisek, P. (2014). Deliberation and commitment in the premotor and primary motor cortex during dynamic decision making. Neuron, 81(6):1401-1416.

Van Vugt, B., Dagnino, B., Vartak, D., Safaai, H., Panzeri, S., Dehaene, S., and Roelfsema, P. R. (2018). The threshold for conscious report: Signal loss and response bias in visual and frontal cortex. Science, 360(6388):537542. 
Vila-Vidal, M., Capouskova, K., Atasoy, S., Kringelbach, M. L., and Deco, G. (2020). Uncovering the spatiotemporal scales of common neuro-mental constructs: Comment on "Is temporo-spatial dynamics the "common currency' of brain and mind? In Quest of 'Spatiotemporal Neuroscience' " by Georg Northoff et al. Physics of Life Reviews, 33:64-66.

Wang, N., Zhang, L., and Liu, G. (2015). EEG-based research on brain functional networks in cognition. Bio-Medical Materials and Engineering, 26(s1):S1107-S1114.

Wang, X., Zhen, Z., Song, Y., Huang, L., Kong, X., and Liu, J. (2016). The hierarchical structure of the face network revealed by its functional connectivity pattern. Journal of Neuroscience, 36(3):890-900.

Wang, X. J. (2002). Probabilistic decision making by slow reverberation in cortical circuits. Neuron, 36(5):955-968.

Zhen, Z., Fang, H., and Liu, J. (2013). The Hierarchical Brain Network for Face Recognition. PLoS ONE, 8(3):59886. 


\section{Supplementary Information}

\section{S1 Spectral power estimation with wavelet and continuous multitaper methods}

Although we chose to use multitaper estimation with DPSS as the main technique to obtain our estimates (used in previous studies such as (Hipp et al., 2012; Lopez-Persem et al., 2020)), we decided to compare the performance of this method with the more widely used wavelet transform method (Rey et al., 2014; Arnulfo et al., 2015; Siebenhühner et al., 2020). Spectral estimation on the time-frequency domain is limited by the fundamental Gabor (aka. Gabor-Heisenberg) uncertainty principle $\left(\Delta t \Delta f \geq \frac{1}{2}\right)$, where where $\Delta t$ and $\Delta f$ represent the uncertainties in time and frequency (Gabor, 1946). In this context, Morlet wavelets are designed to be almost optimal with respect to the previous inequality, yielding the best possible time-frequency resolution, with $\Delta t \Delta f \approx 0.883$ (Morlet et al., 1982; Russell and Han, 2016; Cohen, 2019).

On the other hand, continuous multitaper estimation is designed to perform estimates with reduced bias with respect to true spectral content. This is achieved at the expense of temporal and/or frequency resolution. In particular, this method obtains multiple independent estimates of the same sample, projecting the data onto orthogonal functions or tapers (DPSS, in our case). The maximum number of independent tapers $(K)$ is given by $K=\Delta t \Delta f-1$. In our case, we adapted the parameters to increase the number of tapers with increasing frequency, as the signal-to-noise ratio becomes lower (see Methods, section 2.7).

In the process to detect local neural activity, we compared continuous multitaper and wavelet power estimation in two task-relevant regions from subject 1 . In general, continuous multitaper estimation yielded smoother estimates, as expected. Fig. S1 (top and middle rows) compared spectrograms obtained with both techniques in a contact placed in V1/V2 and in a contact placed in the middle portion (anterior-posterior axis) of the MTG (mMTG). Continuous multitaper estimation revealed consistent high-gamma activity $(64-256 \mathrm{~Hz})$ from 300 to $600 \mathrm{~ms}$ after stimulus presentation in V1/V2, that was only barely detected by wavelet estimation (64-128 Hz, 400 to $500 \mathrm{~ms}$ ). In the mMTG, the continuous multitaper spectrogram revealed more diffuse high-gamma activity $(64-128 \mathrm{~Hz})$ showing a special consistency from 500 to $800 \mathrm{~ms}$ after stimulus presentation. In contrast, those activations were not captured by wavelet estimation.

When averaging across trials, the high temporal resolution of wavelet estimation might cancel out high-frequency activations that are not homogeneously time-locked to the stimulus. To assess whether reduced sensitivity 
of wavelet estimation to high-frequency activity resulted from this effect, we also low-pass filtered the power estimates below $8 \mathrm{~Hz}$ before obtaining median spectrograms across trials (Fig. S1 bottom row). With this approach, high-gamma activations in V1/V2 revealed higher consistency and became comparable to those obtained with continuous multitaper estimation (64$256 \mathrm{~Hz}$ ) from 400 to $500 \mathrm{~ms}$. In contrast, high-gamma activations in the mMTG could not be recovered via wavelet esimation.

Hence, qualitative comparison of continuous multitaper and wavelet power estimation in two task-relevant regions from subject 1 (V1/V2 and mMTG) proved the multitaper method to be more sensitive to sustained high-frequency activations, while fast and highly variable fluctuations across trials tend to be attenuated. In particular, low-amplitude temporally diffuse high-gamma activity $(64-128 \mathrm{~Hz})$ in mMTG was found between 500 to $800 \mathrm{~ms}$ after stimulus presentation by the multitaper method, and, conversely, it could not be detected using the wavelet transform. Another caveat when combining temporally-resolved methods such as the wavelet transform with trial averaging is that small temporal misalignments in power activations across trials might result in these activations cancelling out in the average spectrogram. As a result, stimulus-locked events that occur within a certain temporal window but are not strictly locked to a time point, might remain invisible to exploration. This limitation can be partially overcome by low-pass filtering power estimates (i.e. temporally smoothing) before averaging across trials, as seen in Fig. S1 (bottom, left).

In conclusion, the choice of either method is highly dependent on the research question at hand. In our case, multitaper estimation served the purpose of finding robust task-relevant activations, while cancelling out spurious short-lasting fluctuations. However, in other situations (for instance, when exploring HFO activity, (see (Arnulfo et al., 2015) for an example), wavelet-derived methods might be preferable to capture short transient effects. 

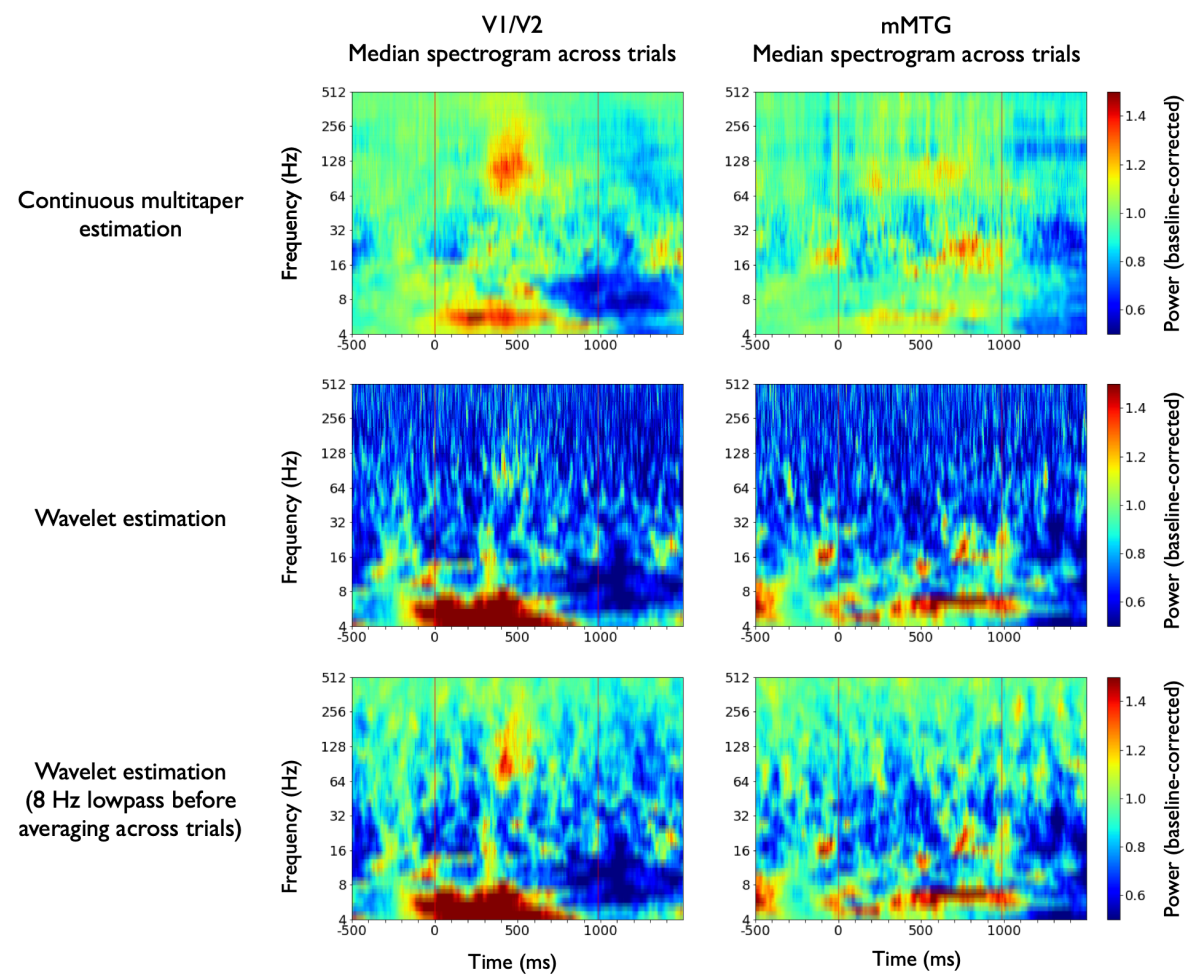

Figure S1: Continuous multitaper and wavelet spectral estimations for two exemplary task-relevant recording sites from subject 1 . In general, multitaper spectrograms tend to be smoother and fast fluctuations are attenuated. In $\mathrm{V} 1 / \mathrm{V} 2$ continuous multitaper estimation revealed consistent high-gamma activity $(64-256 \mathrm{~Hz})$ from 300 to $600 \mathrm{~ms}$ after stimulus presentation in V1/V2 (top left). These activations emerged, although less prominently with wavelet estimation $(64-128 \mathrm{~Hz}, 400$ to $500 \mathrm{~ms}$, middle left). Low-pass filtering the wavelet power estimates below $8 \mathrm{~Hz}$ before obtaining the median spectrogram across trials turned to be more sensitive to these activations, due to potential time misalingments that were thus smoothed (bottom left). In the mMTG (middle portion of the MTG) the continuous multitaper spectrogram revealed diffuse high-gamma activity (64$128 \mathrm{~Hz}$ ) with a special consistency from 500 to $800 \mathrm{~ms}$ after stimulus presentation. These activations were not captured by wavelet estimation. They also remained undetected after low-pass filtering the initial wavelet power estimates below $8 \mathrm{~Hz}$. 


\section{Stimulus-locked global effects}

A
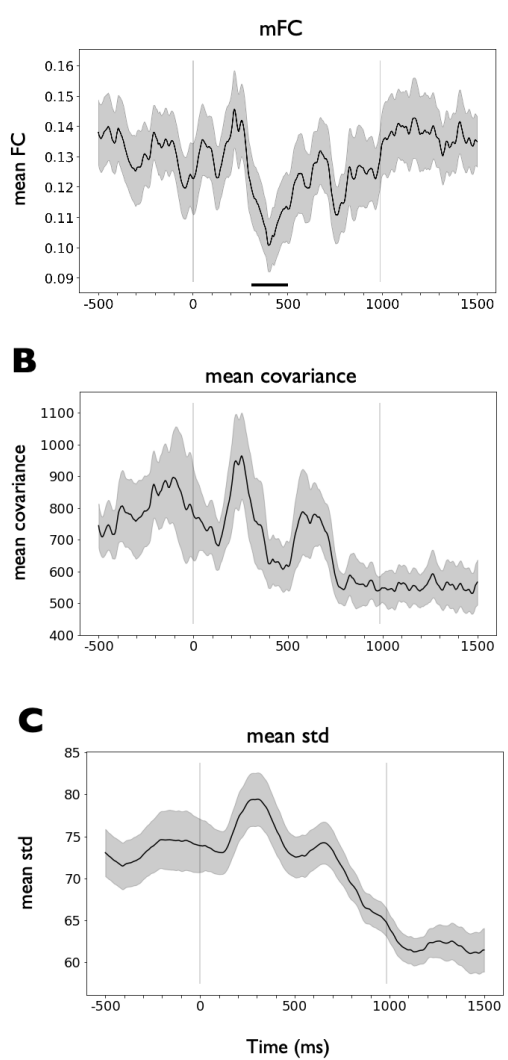

Subject 2
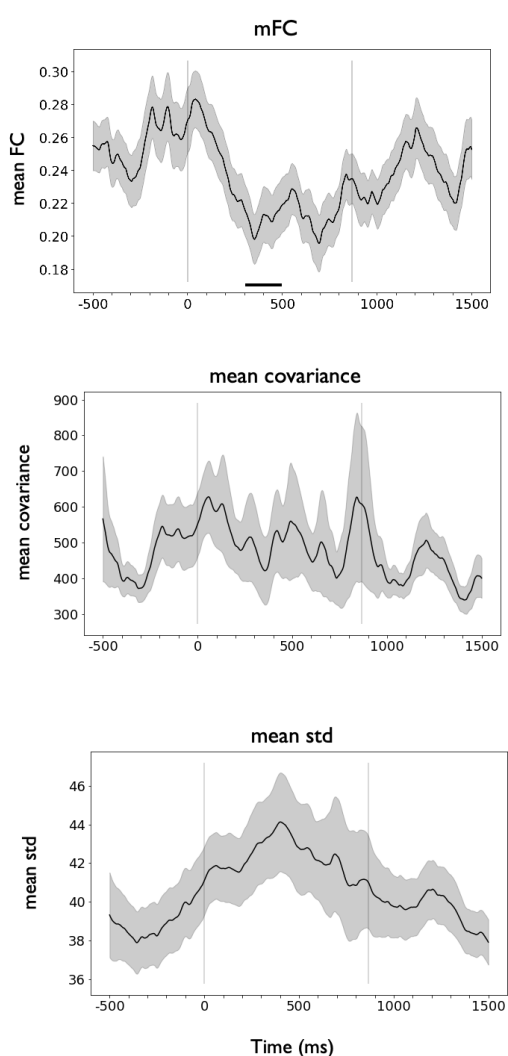

Figure S2: Dissection of the stimulus-locked global effects measured with $\mathrm{mFC}$ into its two components. (A) Time course of the mean functional connectivity (mFC). Median \pm SEM across all trials aligned to stimulus onset. See Methods, section 2.8, for details. Vertical dark lines indicate the stimulus onset and offset times, respectively. A significant decrease in the $\mathrm{mFC}$ associated to stimulus presentation was found in both subjects with respect to their pre-stimulus mean $\mathrm{mFC}$ value (300-500 ms, marked with black bars, Ranksum test applied across trials on the mean of the $\mathrm{mFC}$ in the baseline period and the 300-500 ms window, $P<0.05$ ). (B) Average covariance across all pairs of selected GM contacts. (C) Average standard deviation across all GM contacts. 

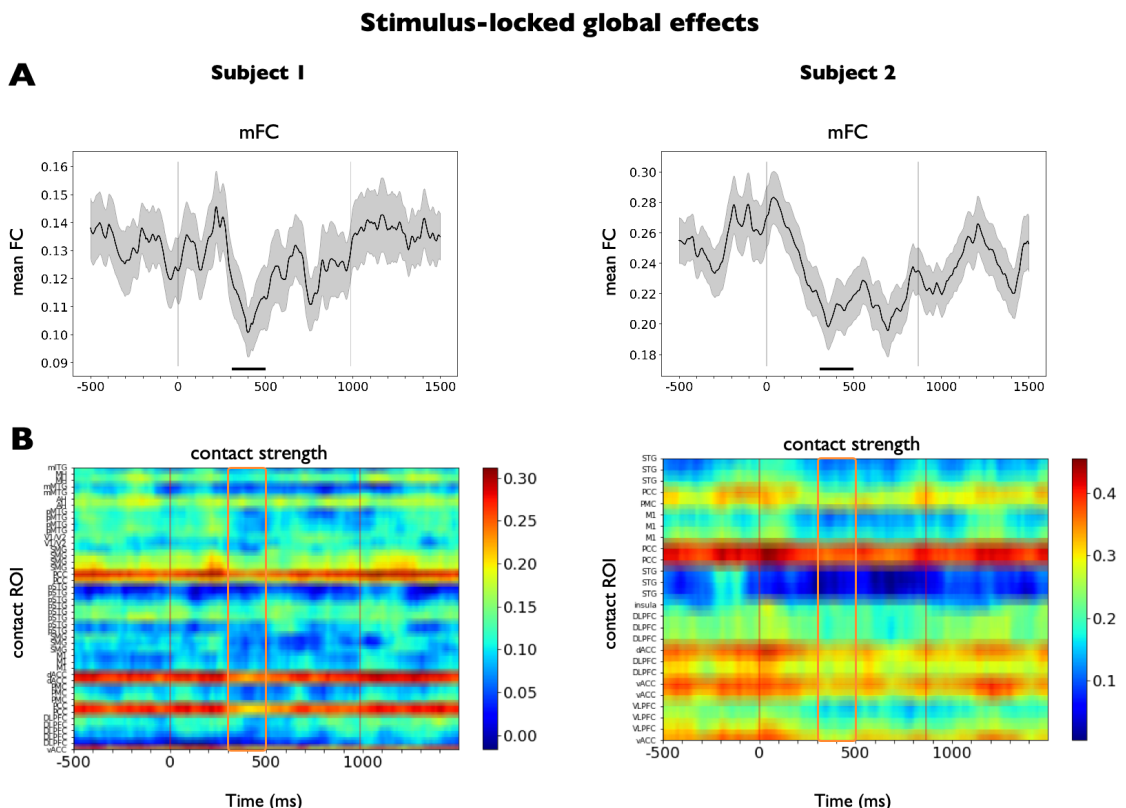

C
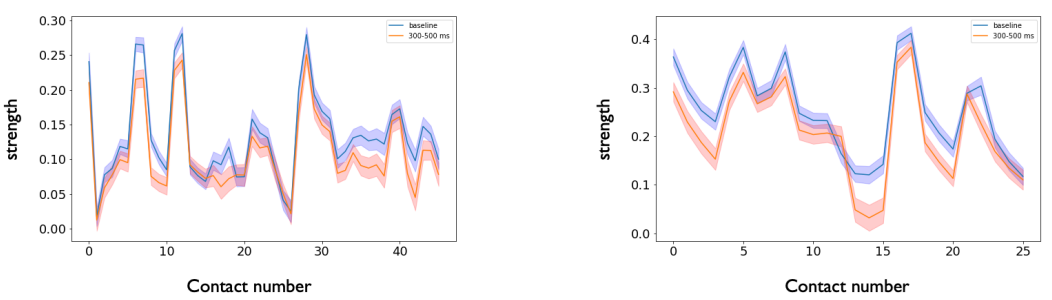

Figure S3: Connectivity fluctuations and contact strength across the task measured with FC. (A) Time course of the mean functional connectivity (mFC). Median \pm SEM across all trials aligned to stimulus onset. See Methods, section 2.8, for details. Vertical dark lines indicate the stimulus onset and offset times, respectively. A significant decrease in the $\mathrm{mFC}$ associated to stimulus presentation was found in the two subjects with respect to their pre-stimulus mean mFC value (300-500 ms, marked with black bars, Ranksum test applied across trials on the mean of the mFC in the baseline period and the 300-500 ms window, $P<0.05$ ). (B) Contact connectivity strength (average of the seed-based connectivity against its matching GM contact pairs). The period 300-500 ms is marked in red. (C) Average contact strength in the pre-stimulus baseline period (from -500 to -100 $\mathrm{ms}$, blue) and the marked stimulus epoch (300-500 ms). The decay in FC is a generalized effect across the implantation scheme of both subjects. Median \pm SEM across all trials. 




$\mathbf{A}$

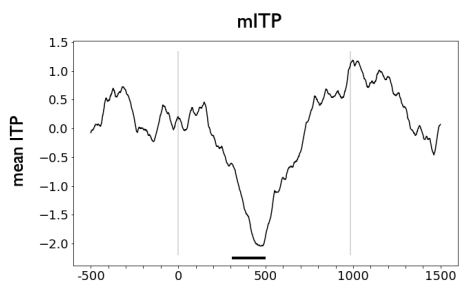

B

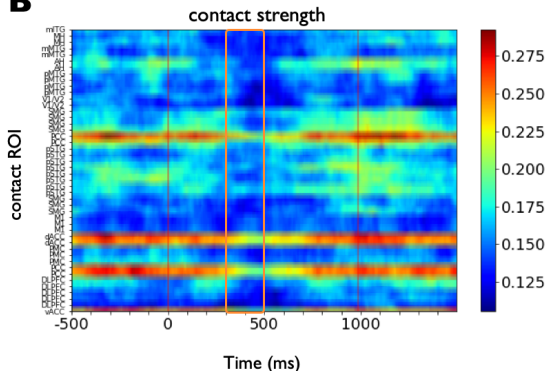

C

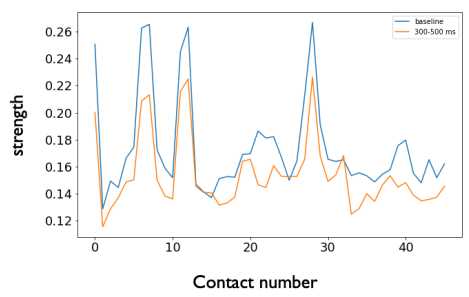

Subject 2



contact strength
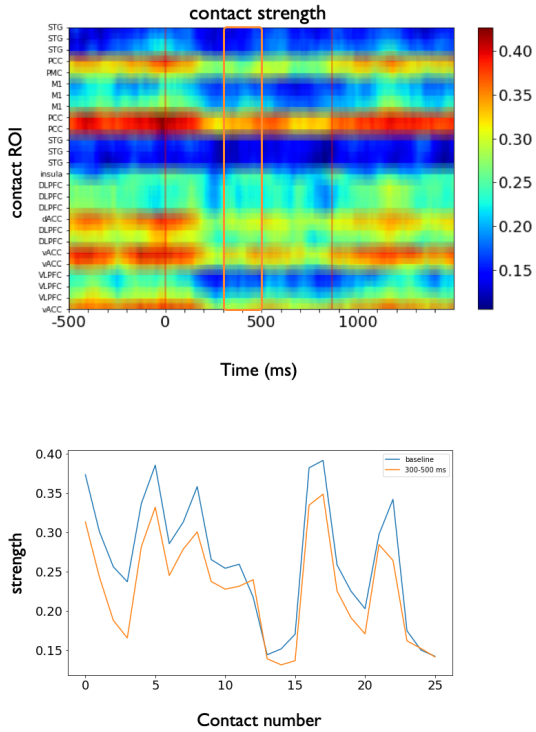

Figure S4: Connectivity fluctuations and contact strength across the task measured with ITP. (A) Time course of the mean inter-trial phase coherence (mITP). See Methods, section 2.8, for details. Vertical dark lines indicate the stimulus onset and offset times, respectively. A significant decrease in the mITP associated to stimulus presentation was found in the two subjects with respect to their pre-stimulus mean mFC value $(300-500$ ms, marked with black bars, Ranksum test applied across trials on the mean of the mFC in the baseline period and the 300-500 ms window, $P<0.05$ ). (B) Contact connectivity strength (average of the seed-based connectivity against its matching GM contact pairs). The period 300-500 ms is marked in red. (C) Average contact strength in the pre-stimulus baseline period (from -500 to $-100 \mathrm{~ms}$, blue) and the marked stimulus epoch (300-500 ms). The decay in ITP is a generalized effect across the implantation scheme of both subjects. 

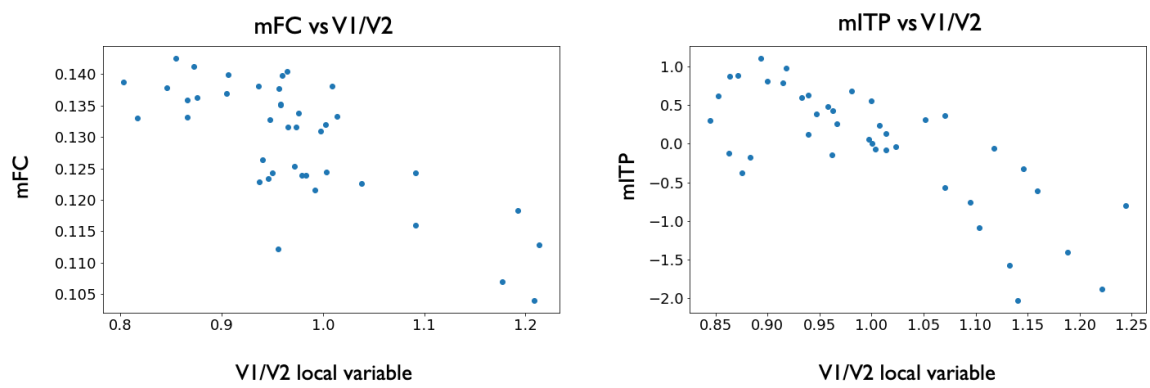

B

Motor-locked local-global in subject 2
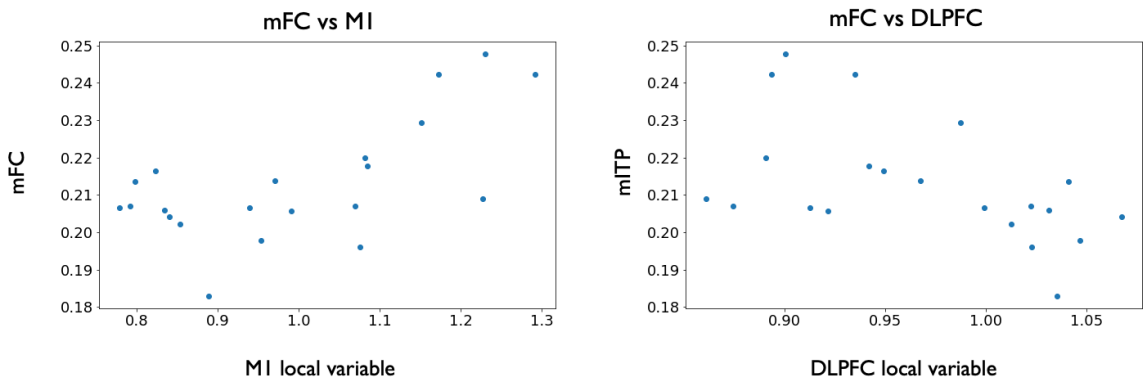

Figure S5: Exemplary scatter plots showing the association between local and global variables that were found to be significant. (A) Scatter plots in the stimulus-locked setting for subject 1 showing the association between $\mathrm{mFC}$ and a contact in V1/V2 (left), and between mITP and a contact in V1/V2 (right). (B) Scatter plots in the motor-locked setting for subject $2 \mathrm{~s}$ howing the association between $\mathrm{mFC}$ and a contact in M1 (left), and between $\mathrm{mFC}$ and a contact in DLPFC (right). All variables have been downsampled by a factor of 100 , to avoid autocorrelation effects. 